\title{
Non-adiabatic effects in long-pulse mixed-field orientation of a linear polar molecule
}

\author{
Juan J. Omiste* and Rosario González-Férezt \\ Instituto Carlos I de Física Teórica y Computacional, and Departamento de Física Atómica, \\ Molecular y Nuclear, Universidad de Granada, 18071 Granada, Spain
}

(Dated: July 10, 2018)

\begin{abstract}
We present a theoretical study of the impact of an electrostatic field combined with non-resonant linearly polarized laser pulses on the rotational dynamics of linear molecules. Within the rigid rotor approximation, we solve the time-dependent Schrödinger equation for several field configurations. Using the OCS molecule as prototype, the field-dressed dynamics is analyzed in detail for experimentally accessible static field strengths and laser pulses. Results for directional cosines are presented and compared to the predictions of the adiabatic theory. We demonstrate that for prototypical field configuration used in current mixed-field orientation experiments, the molecular field dynamics is, in general, non-adiabatic, being mandatory a time-dependent description of these systems. We investigate several field regimes identifying the sources of non-adiabatic effects, and provide the field parameters under which the adiabatic dynamics would be achieved.
\end{abstract}

PACS numbers: $37.10 . \mathrm{Vz}, 33.80 .-\mathrm{b}, 33.57 .+\mathrm{c}, 42.50 . \mathrm{Hz}$

\section{INTRODUCTION}

The creation of directional states of molecules represents an important tool to control and tailor the rotational degree of freedom. When a molecule is oriented the molecular fixed axes are confined along laboratory fixed axes and its dipole moment is pointing in a particular direction. Experimentally, the availability of oriented molecules provides a wealth of interesting applications in a variety of molecular sciences, such as in chemical reaction dynamics [1 5], photoelectron angular distributions [6 8], or high-order harmonic generation [9, 10].

Due to this broad interest, special efforts have been undertaken to create samples of oriented molecules and techniques based in the application of inhomogeneous 1, 11], and homogeneous [12 16 electric fields as well as homogeneous magnetic fields [17] have been used. A major breakthrough came with the proposal by Friedrich and Herschbach [18, 19] of enhancing the orientation of polar molecules by exposing them to combined weak electrostatic and strong non-resonant radiative fields. This theoretical prediction was done within an adiabatic picture assuming that the switching on time of the laser pulse is larger than the molecular rotational period. For linear molecules, a linearly polarized laser field produces a double-well potential along the polarization direction. In the pendular limit, this double-well potential contains nearly degenerate pairs of states with opposite parity forming tunneling doublets. If the molecules possess a permanent electric dipole moment, a strong pseudo-firstorder Stark effect is induced by coupling the tunneling doublets with an additional electrostatic field. Due to this coupling, the two levels in a pendular doublet are efficiently oriented but with their effective electric dipole

\footnotetext{
* omiste@ugr.es

$\dagger$ rogonzal@ugr.es
}

moments pointing in opposite directions. As a consequence of this oriented and antioriented states pairing, the orientation is small in a molecular ensemble with the population thermally distributed. Therefore, the first experimental measures of the orientation of a molecular beam were indeed reduced to small values [20, 21]. A significant improvement was gained by using a quantumstate selected molecular beam, which allowed the creation of unprecedented degree of orientation for complex asymmetric tops [22 24]. A first theoretical study of the mixed-field orientation experiment of asymmetric top molecules, pointed out that a fully adiabatic description of the process does not reproduce the experimental observations [25].

Recently, we have experimentally and theoretically investigated the mixed-field orientation of the carbonyl sulfide molecule [26]. Our analysis has proven that a timedependent description of the mixed-field orientation process is required to explain the experimental results. We have shown how the non-adiabatic coupling of the levels forming the quasi-degenerate doublets as the laser intensity is increased, gives rise to the reduction of the orientation and, therefore, to the disagreement with the predictions of the adiabatic theory [18, 19]. Herein, we provide a detailed theoretical analysis on the dynamics of a linear molecule exposed to an electrostatic field combined with a non-resonant laser pulse. In the framework of the rigid rotor approximation, we solve the timedependent Schrödinger equation using experimental field configurations, i.e., a Gaussian laser pulse and a weak electrostatic field that is turned on at constant speed. As prototype example, we consider the carbonyl sulfide molecule (OCS). For several rotational states, we investigate the mixed-field orientation dynamics under different field-configurations by varying either the laser peak intensity, the duration of the Gaussian pulse, the dc field strength or the angle between both fields. Hence, we demonstrate that for some field configurations, the fielddressed dynamics is non-adiabatic and provide a detailed 
account of the sources of non-adiabaticity and the field regimes at which they appear. For parallel fields, the dynamics is characterized by the population transfer between adiabatic states when the pendular doublets are formed. Whereas for non-parallel fields, we encounter additional non-adiabatic effects when the states from the same $J$-manifold, having now the same symmetry, are driven apart as the laser intensity is increased on the weak field regime. For different field configurations, we identify and discuss the experimental conditions needed to achieve an adiabatic molecular dynamics.

The paper is organized as follows: In Sec. II we describe the Hamiltonian of the system and its symmetries for various field configurations. The results for the energy, alignment and orientation predicted by the adiabatic theory are analyzed in Sec. III In Sec. IV we focus on the molecular dynamics when the fields are parallel. In particular, we explore how the time-dependent orientation varies as the field parameters are modified, and indicate the experimental conditions under which an adiabatic orientation would be achieved. A similar study is performed for tilted fields in Sec. V] where we show that the conditions for an adiabatic mixed-field orientation are more difficult to fulfill. In Sec. VI] we assume that once the pulse is turned on its peak intensity is kept constant, and investigate the dynamics in this regime. In Sec. VII. first the laser pulse is switched on and then an electric field is applied. In this field configuration, we analyze the orientation of the ground state and provide the field parameters for an adiabatic orientation. The conclusions are given in Sec. VIII

\section{THE HAMILTONIAN OF A LINEAR RIGID ROTOR IN EXTERNAL FIELDS}

We consider a polar linear molecule exposed to an homogeneous static electric field and a non-resonant linearly polarized laser pulse. The field configuration is illustrated in Fig. 1 the polarization of the laser field lies along the $Z$-axis of the laboratory fixed frame (LFF) $(X, Y, Z)$, and the dc field is contained in the $X Z$-plane forming an angle $\beta$ with the $Z$-axis. The $z$-axis of the molecule fixed frame $(x, y, z)$ is defined by the permanent dipole moment $\boldsymbol{\mu}$ of the molecule. These two frames are related by the Euler angles $\Omega=(\theta, \phi)$, cf Fig. 1 . The description of this system is done within the rigid rotor approximation, assuming that the vibrational and electronic dynamics are not affected by the fields. Thus, the rigid rotor Hamiltonian reads

$$
H(t)=H_{r}+H_{\mathrm{S}}(t)+H_{\mathrm{L}}(t),
$$

where $H_{r}$ is the field-free Hamiltonian

$$
H_{r}=B \mathbf{J}^{2}
$$

with $\mathbf{J}$ being the total angular momentum operator and $B$ the rotational constant. The terms $H_{\mathrm{S}}(t)$ and $H_{L}(t)$ stands for the interactions with the static and laser fields, respectively.

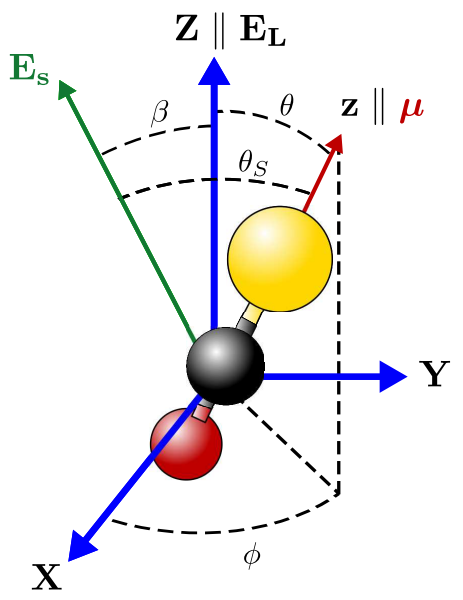

FIG. 1. Laboratory fixed coordinate, Euler angles, schematic field configuration and the OCS molecule.

The dipole coupling with the static field reads

$$
H_{\mathrm{S}}(t)=-\boldsymbol{\mu} \cdot \mathbf{E}_{\mathrm{s}}(t)=-\mu \mathrm{E}_{\mathrm{s}}(t) \cos \theta_{\mathrm{s}}
$$

with $\mathbf{E}_{\mathrm{s}}(t)=\mathrm{E}_{\mathrm{s}}(t)(\sin \beta \hat{X}+\cos \beta \hat{Z})$, and $\mathrm{E}_{\mathrm{s}}(t)$ being the electrostatic field strength. The angle between the dipole moment $\boldsymbol{\mu}$ and this field is $\theta_{\mathrm{s}}$, cf Fig. 1, and $\cos \theta_{\mathrm{s}}=$ $\cos \beta \cos \theta+\sin \beta \sin \theta \cos \phi$.

We consider a non-resonant laser field linearly polarized along the $Z$-axis of the LFF, $\mathbf{E}_{\mathrm{L}}(t)=$ $\mathrm{E}_{0} g(t) \cos (2 \pi \nu t) \hat{Z}$, with $\nu$ being its frequency, $\mathrm{E}_{0}$ the peak field strength, and $g(t)$ the pulse envelope. Assuming that $\nu^{-1}$ is much shorter than the pulse duration and the rotational period, we average over the rapid oscillations of the non-resonant field. This causes the coupling of this field with the permanent dipole moment to vanish [27, 28]. Thus, the non-resonant laser field molecule interaction can be written as

$$
H_{\mathrm{L}}(t)=-\frac{\mathrm{I}(t)}{2 c \epsilon_{0}} \Delta \alpha \cos ^{2} \theta
$$

where $\Delta \alpha$ is the polarizability anisotropy, $\mathrm{I}(t)$ is the intensity of the laser, $c$ is the speed of light and $\epsilon_{0}$ is the dielectric constant. Note that we have neglected the term $-\alpha_{\perp} \mathrm{I}(t) / 2 c \epsilon_{0}$, which represents only a shift in the energy.

In this work, the field configurations are chosen based on the mixed-field orientation experiments [22, 24, 26]. Initially, the molecule is in field-free space, then the electrostatic field is switched on, and its strength is increased linearly with time. At $t=-T_{0}$ the maximum strength is achieved and kept constant afterwards. This time $T_{0}$ is chosen long enough to ensure the adiabaticity of this turning-on process. For the laser pulse, we use a linearly polarized Gaussian pulse with a full width half maximum (FWHM) $\tau$ on the nanosecond range. The intensity is given by $\mathrm{I}(t)=\mathrm{I}_{0} \exp \left(-t^{2} / 2 \sigma^{2}\right)$, with $\mathrm{I}_{0}$ being the peak 
Table I. Action of the symmetry operations on the Euler angles.

\begin{tabular}{lll}
\hline \hline & Transformations & \\
\hline Operation & $\phi$ & $\theta$ \\
\hline $\mathcal{E}$ & $\phi \rightarrow \phi$ & $\theta \rightarrow \theta$ \\
$\sigma_{X Z}$ & $\phi \rightarrow 2 \pi-\phi$ & $\theta \rightarrow \theta$ \\
$\mathcal{C}_{X}(\pi)$ & $\phi \rightarrow 2 \pi-\phi$ & $\theta \rightarrow \pi-\theta$ \\
$\mathcal{C}_{\perp Z}^{\alpha}(\pi)$ & $\phi \rightarrow 2 \alpha-\phi$ & $\theta \rightarrow \pi-\theta$ \\
$\mathcal{C}_{Z}(\delta)$ & $\phi \rightarrow \phi+\delta$ & $\theta \rightarrow \theta$ \\
\hline \hline
\end{tabular}

intensity, and $\sigma$ is related with the FWHM $\tau=2 \sqrt{2 \ln 2} \sigma$. Numerically, the non-resonant laser field is turned on in such a way that the interaction due to this field is much weaker than coupling with the dc field.

The eigenstates of the field-free Hamiltonian (2) are the spherical harmonics $Y_{J M}(\Omega)$, with $J$ and $M$ being the rotational and magnetic quantum numbers, respectively. Note that $M$ is the projection of the total angular momentum $\mathbf{J}$ on the LFF $Z$-axis. The field-free Hamiltonian (2) belongs to the $\mathrm{SO}(3)$ group because the operator $\overline{\mathbf{J}}^{2}$ remains unaltered under any rotation. In the presence of the external fields, the symmetries of the rotational Hamiltonian (1) are significantly reduced. The operations describing the symmetries of the field-dressed Hamiltonian are collected in Table I For a static field, the symmetry group is reduced to arbitrary rotations around the field axis and the identity $\left\{\mathcal{E}, \mathcal{C}_{\mathbf{E}_{\mathbf{s}}}(\delta)\right\}$. If only a linearly polarized laser field is applied, the Hamiltonian is invariant under arbitrary rotations around $Z$ axis and two-fold rotations around any axis perpendicular to the $Z$-axis, and the symmetry group is compound by $\left\{\mathcal{E}, \mathcal{C}_{Z}(\delta), \mathcal{C}_{\perp Z}(\pi)\right\}$. For parallel fields, the elements of the symmetry group are the identity $\mathcal{E}$, arbitrary rotations around the fields $\mathcal{C}_{Z}(\delta)$ and the reflection in any plane containing the fields. For a given $|M|$-value, the parity under the reflection on one of these planes defines two irreducible representations. Since the selection of this plane is not unique, then the states with $M \neq 0$ are doubly degenerated. Note that $M$ remains as a good quantum number for these three field configurations with $\beta=0^{\circ}$. For non-perpendicular and non-collinear fields, i.e., $\beta \neq 0^{\circ}$ and $\beta \neq 90^{\circ}$, the Hamiltonian is invariant under the identity $\mathcal{E}$ and the reflection on the $X Z$ plane containing the fields $\sigma_{X Z}$. This group $\left\{\mathcal{E}, \sigma_{X Z}\right\}$ has only two irreducible representations characterized by the parity with respect to this reflection $\sigma_{X Z}$, and the functions belonging to the even and odd representations are $\Psi_{J M}^{e / o}(\Omega)=\left(Y_{J M}(\Omega)+(-1)^{\kappa} Y_{J-M}(\Omega)\right) / \sqrt{2}$, with $\kappa$ given in Table II. If the fields are perpendicular, $\beta=90^{\circ}$, the two-fold rotation around the static field $\mathcal{C}_{X}(\pi)$ is also a symmetry operation. Thus, the symmetry group is formed by $\left\{\mathcal{E}, \sigma_{X Z}, \mathcal{C}_{X}(\pi)\right\}$ has four irreducible representations labeled by the parity with respect to the transformations $\sigma_{X Z}$ and $\mathcal{C}_{X}(\pi)$. The properly symmetrized functions for these four irreducible representations are $\Psi_{J M}^{e / o, e / o}(\Omega)=\left(Y_{J M}(\Omega)+(-1)^{\epsilon} Y_{J-M}(\Omega)\right) / \sqrt{2}$, the possible values of $\epsilon$ are collected in Table II
Table II. For the $\beta=90^{\circ}$ and $0^{\circ}<\beta<90^{\circ}$ field configurations, values of the parameters $\kappa$ and $\epsilon$ for the wave functions with the correct symmetry for each irreducible representation.

\begin{tabular}{|c|c|c|c|c|c|}
\hline \multicolumn{2}{|c|}{$\beta \neq 90^{\circ}$} & \multicolumn{3}{|c|}{$\beta=90^{\circ}$} & \multirow[b]{2}{*}{$\mathcal{C}_{X}$} \\
\hline$\kappa$ & $\sigma_{X Z}$ & $\epsilon$ & $J+M$ & $\sigma_{X Z}$ & \\
\hline$M$ & even & $\bar{M}$ & even & even & even \\
\hline$M+1$ & odd & $M$ & odd & even & odd \\
\hline & & $M+1$ & odd & odd & even \\
\hline & & $M+1$ & even & odd & even \\
\hline
\end{tabular}

The time-dependent Schrödinger equation associated to the Hamiltonian (1) is solved by means of a secondorder split-operator technique 29], combined with the discrete-variable and finite-basis representation methods for the angular coordinates [30 33]. For reasons of addressability, we will label the time-dependent states as $|J, M, l\rangle^{0}$ and $|J, M, l\rangle$ for $\beta=0^{\circ}$ and $0^{\circ}<\beta<90^{\circ}$, respectively, with $l=e$ and $o$ indicating even or odd parity with respect to the $X Z$-plane. The labels $J$ and $M$ refer to field-free quantum number to which they are adiabatically connected. Note that the labeling of the states depends on the way the fields are turned on 34 ]. The time-dependent wave function depends on the time $t$, the peak intensity $\mathrm{I}_{0}$, the FWHM $\tau$, the electrostatic field strength $\mathrm{E}_{\mathrm{s}}$, and the angle $\beta$. For the sake of simplicity, we have not made explicit these dependences, but the field configuration is clearly indicated through the text.

To get a better physical insight on the field-dressed dynamics, the time-dependent results will be compared to those from the adiabatic theory. For this system, we take the adiabatic limit by using a constant electrostatic field $\mathrm{E}_{\mathrm{s}}$ and constant laser intensity $\mathrm{I}$ in the Hamiltonian (1). The time-independent Schrödinger equation associated to this Hamiltonian is solved by expanding the wave function in a basis that respects the symmetries. The adiabatic states are labeled as $|J, M, l\rangle_{\mathrm{p}}^{0}$ and $|J, M, l\rangle_{\mathrm{p}}$ for $\beta=0^{\circ}$ and $0^{\circ}<\beta<90^{\circ}$, respectively, and we have not made explicit their dependence on the field parameters.

The field-dressed eigenfunctions of this timeindependent Hamiltonian form a basis, which is used to analyze the time-dependent wave function $|\gamma\rangle$ by means of the following expansion

$$
|\gamma\rangle=\sum_{i=1}^{N} C_{i}(t)\left|\gamma_{i}\right\rangle_{\mathrm{p}}
$$

with $C_{i}(t)=\left\langle\gamma \mid \gamma_{i}\right\rangle_{\mathrm{p}}, \gamma$ and $\gamma_{i}$ including all the labels identifying these levels. For computational reasons, we have only considered the lowest-lying $N$ adiabatic levels, and always ensured that the contributions of highly excited states are negligible. Let us remark that for each time $t$, the expansion of the wave function is performed in a different adiabatic basis obtained by solving the timeindependent Schrödinger equation using the static field strength and laser intensity at time $t$, i.e., $\mathrm{E}_{\mathrm{s}}(t)$ and $\mathrm{I}(t)$. 


\section{RESULTS IN THE ADIABATIC LIMIT}

In this work, we use the OCS molecule (see fig. 1) as benchmark to illustrate our results. The rotational constant of OCS is $B=0.209 \mathrm{~cm}^{-1}$, the permanent dipole moment $\mu=0.709 \mathrm{D}$ and the polarizability anisotropy $\Delta \alpha=4.04 \AA^{3}$.

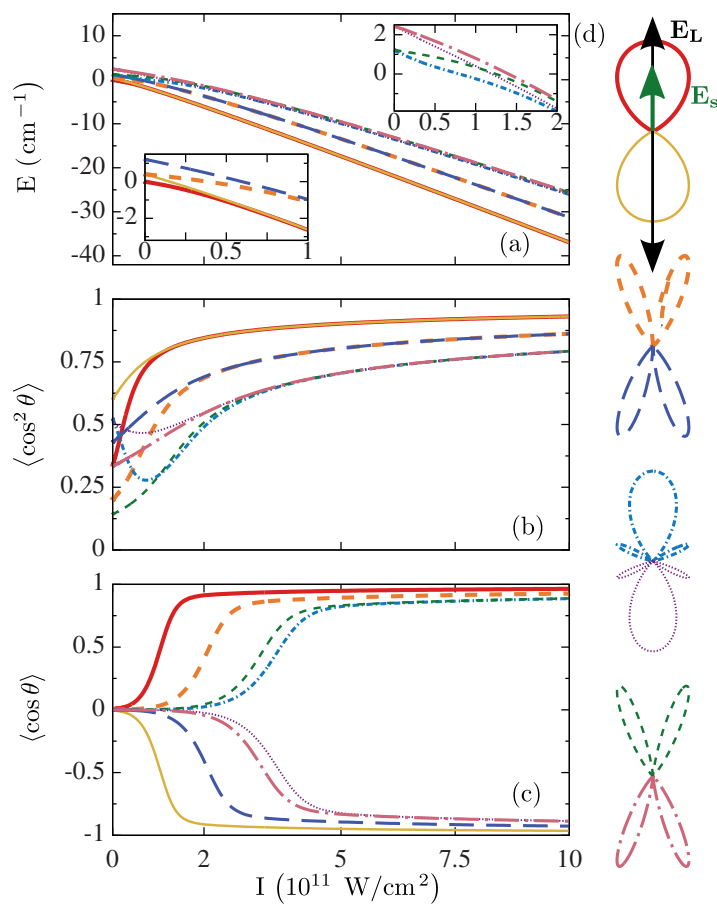

FIG. 2. (Color online) Adiabatic results for the (a) energy, expectation values (b) $\left\langle\cos ^{2} \theta\right\rangle$ and (c) $\langle\cos \theta\rangle$ as a function I of the adiabatic states $|0,0, e\rangle_{\mathrm{p}}^{0}$ (red thick solid), $|1,0, e\rangle_{\mathrm{p}}^{0}$ (gold thin solid), $|1,1, e\rangle_{\mathrm{p}}^{0}$ (orange thick short-dashed), $|2,1, e\rangle_{\mathrm{p}}^{0}$ (dark blue long-dashed), $|2,0, e\rangle_{\mathrm{p}}^{0}$ (blue dot-short-dashed), $|3,0, e\rangle_{\mathrm{p}}^{0}$ (purple dotted), $|2,2, e\rangle_{\mathrm{p}}^{0}$ (green thin short-dashed), and $|3,2, e\rangle_{\mathrm{p}}^{0}$ (pink dot-long-dashed). The insets show the relevant energy and intensity ranges where the formation of the near-degenerate doublets occurs. (d) Polar plots of the square of their wave functions at $\mathrm{I}=10^{12} \mathrm{~W} / \mathrm{cm}^{2} . \mathrm{E}_{\mathrm{s}}=300 \mathrm{~V} / \mathrm{cm}$ and $\beta=0^{\circ}$ for all data.

We start by analyzing the adiabatic limit. We restrict this study to the following eight states: $|0,0, e\rangle_{\mathrm{p}}^{0}$, $|1,0, e\rangle_{\mathrm{p}}^{0},|1,1, e\rangle_{\mathrm{p}}^{0},|2,1, e\rangle_{\mathrm{p}}^{0},|2,0, e\rangle_{\mathrm{p}}^{0},|3,0, e\rangle_{\mathrm{p}}^{0},|2,2, e\rangle_{\mathrm{p}}^{0}$, and $|3,2, e\rangle_{\mathrm{p}}^{0}$. For $\beta=0^{\circ}$, they adiabatically correspond to the states forming the four first doublets. Note that they well represent the main physical features observed in the overall molecular dynamics, and similar behavior and properties are, therefore, obtained for states in other irreducible representations.

For $\mathrm{E}_{\mathrm{s}}=300 \mathrm{~V} / \mathrm{cm}$ and $\beta=0^{\circ}$, the energies and the expectation values $\left\langle\cos ^{2} \theta\right\rangle$ and $\langle\cos \theta\rangle$ of these levels are plotted versus the laser intensity in Fig. 2 (a), (b) and (c), respectively. The weak static field breaks the fieldfree degeneracy in the magnetic quantum number and, as the laser intensity is increased, these states become high field seekers. In the strong laser field regime, once the pendular regime is reached, pairs of quasi-degenerate states with the same symmetry are formed. The insets in this panel show how these doublets appear. The gap in energy in a doublet goes as $\Delta E \approx 2\left|\mu \mathrm{E}_{\mathrm{s} \mathrm{p}}\langle i|\cos \theta| i\rangle_{\mathrm{p}}\right|$, where $\mu_{\mathrm{p}}\langle i|\cos \theta| i\rangle_{\mathrm{p}}$ is the effective dipole moment of the state $|i\rangle_{\mathrm{p}}$ in the doublet, which is of opposite sign for $|j\rangle_{\mathrm{p}}$. Within a doublet, the two levels are characterized by the same hybridization of the angular motion $\left\langle\mathbf{J}^{2}\right\rangle$ and alignment $\left\langle\cos ^{2} \theta\right\rangle$, see Fig. 2 (b). In contrast, they possess opposite orientation $\langle\cos \theta\rangle$, one being oriented and the other antioriented, cf. Fig. 2 (c). This opposite orientation is also illustrated in Fig. 2(d) by the polar plots of the square of their wavefunctions for $\mathrm{I}=10^{12} \mathrm{~W} / \mathrm{cm}^{2}$. The larger is the field-free rotational quantum number of the levels, i. e., their field-free energy, the stronger is the laser intensity needed to achieve a significant orientation. Indeed, the states in the third and fourth doublets are not aligned for $\mathrm{I} \lesssim 2 \times 10^{11} \mathrm{~W} / \mathrm{cm}^{2}$ and, therefore, not oriented. Once the pendular regime is achieved, the orientation of these states $|\langle\cos \theta\rangle|$ approaches to 1 as $I$ is enhanced. If the laser field is sufficiently strong, this adiabatic orientation is independent of the dc field strength, and of the angle between both fields.

\section{RESULTS FOR PARALLEL FIELDS}

In this section, we investigate the rotational dynamics in a parallel configuration: a dc-field of $300 \mathrm{~V} / \mathrm{cm}$ and a Gaussian pulse with FWHM $\tau=10$ ns and several peak intensities. For the ground state $|0,0, e\rangle^{0}$, the expectation value $\langle\cos \theta\rangle$ is presented in Fig. 3 (a) as a function of $\mathrm{I}(t)$ up until the peak intensity $\mathrm{I}_{0}$ is reached. For comparison, the adiabatic results are also shown.

Since the FWHM is 125.31 times larger than the rotational period, one would expect that the rotational dynamics follows the adiabatic limit. However this is not the case, and there are significant discrepancies between the time-dependent and adiabatic results. In contrast to what is predicted by the adiabatic theory, the final orientation decreases as the peak intensity of the laser pulse is increased. For $\mathrm{I}_{0}=2 \times 10^{11} \mathrm{~W} / \mathrm{cm}^{2}$, $\langle\cos \theta\rangle$ initially resembles the adiabatic behavior, but it achieves a maximum value $\langle\cos \theta\rangle=0.899$ for $\mathrm{I}(t)=1.86 \times 10^{11} \mathrm{~W} / \mathrm{cm}^{2}$. For $\mathrm{I}_{0}=5 \times 10^{11} \mathrm{~W} / \mathrm{cm}^{2}$, $10^{12} \mathrm{~W} / \mathrm{cm}^{2}$, and $2 \times 10^{12} \mathrm{~W} / \mathrm{cm}^{2}$, the orientation shows a qualitatively similar but quantitatively different behavior: in the weak laser field regime, $\langle\cos \theta\rangle$ monotonically increases following the adiabatic limit, but for $\mathrm{I}(t) \gtrsim 2 \times 10^{11} \mathrm{~W} / \mathrm{cm}^{2}$ it reaches a plateau behavior being the orientation smaller than the adiabatic value. For instance, $\langle\cos \theta\rangle=0.661$ for $\mathrm{I}(0)=2 \times 10^{12} \mathrm{~W} / \mathrm{cm}^{2}$ whereas the adiabatic value is $\langle\cos \theta\rangle=0.975$.

A first physical insight into the non-adiabatic dynamics could be gained by analyzing the characteristic times of the molecule. When the states in a pendular dou- 

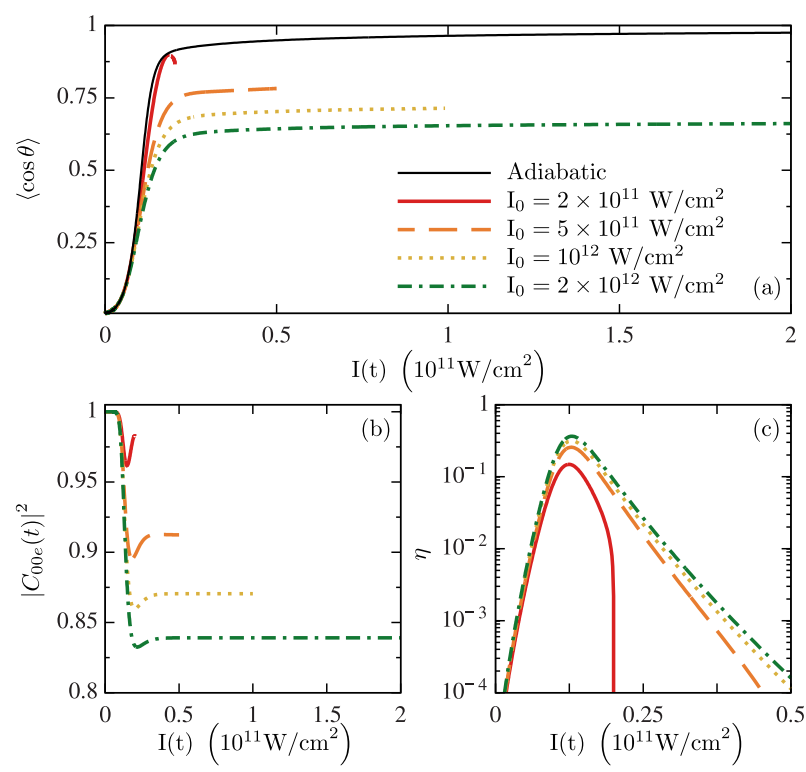

FIG. 3. (Color online) (a) For the ground state, time evolution of the expectation value $\langle\cos \theta\rangle$ as a function of $\mathrm{I}(t)$ for Gaussian pulses of $\tau=10 \mathrm{~ns}$ and peak intensities $\mathrm{I}_{0}=2 \times 10^{11} \mathrm{~W} / \mathrm{cm}^{2}$ (red thick solid), $5 \times 10^{11} \mathrm{~W} / \mathrm{cm}^{2}$ (orange dashed), $10^{12} \mathrm{~W} / \mathrm{cm}^{2}$ (gold dotted) and $2 \times 10^{12} \mathrm{~W} / \mathrm{cm}^{2}$ (green dot-dashed). The adiabatic results for $\langle\cos \theta\rangle$ (thin solid) are also included. (b) The squares of the projections of the time-dependent wave functions onto the adiabatic pendular state $|0,0, e\rangle_{\mathrm{p}}^{0}$. (c) Adiabatic criteria $\eta$ as a function of $\mathrm{I}(t)$. The field configuration is $\mathrm{E}_{\mathrm{s}}=300 \mathrm{~V} / \mathrm{cm}$ and $\beta=0^{\circ}$.

blet are quasi-degenerated, the energy gap between them, $\Delta E \sim 2 \mu \mathrm{E}_{\mathrm{s}}$, defines a time scale of this system [26]. Note that we have assumed $\left.\right|_{\mathrm{p}}\langle i|\cos \theta| i\rangle_{\mathrm{p}}|=|_{\mathrm{p}}\langle j|\cos \theta| j\rangle_{\mathrm{p}} \mid \approx$ 1 , which holds in the strong laser field regime. For $\mathrm{E}_{\mathrm{s}}=300 \mathrm{~V} / \mathrm{cm}$ and $\beta=0^{\circ}$, the energy separation within the first doublet formed by $|0,0, e\rangle_{\mathrm{p}}^{0}$ and $|1,0, e\rangle_{\mathrm{p}}^{0}$ is $\Delta E=6.97 \times 10^{-4} \mathrm{~cm}^{-1}$ giving a time scale of $761.21 \mathrm{ps}$, which is larger than the rotational period $79.8 \mathrm{ps}$. Thus, only long enough pulses compared to this pendular time would ensure an adiabatic orientation of the molecule.

Since the static field strength is so weak, its impact on the rotational dynamics is very small, and before the pulse the levels could be considered as field-free rotor states. As the laser intensity increases, the states are hybridized by the combined action of the both fields, and the doublets of nearly-degenerate states are formed in the strong laser field regime, as it is shown in Fig. 2 (a). When the energy splitting of this pendular doublet approaches the coupling of the two sublevels due to the pseudo-first-order Stark interaction, these states can mix because they have the same symmetry for $\beta \neq 90^{\circ}$. As a consequence, there is a population transfer between the oriented and anti-oriented states, which results in a decrease of the final orientation compared to the adiabatic limit. For this field configuration, the dynamics can be analyzed by means of the adiabatic states forming this pendular doublet, because their couplings to states in neighboring doublets is much smaller than the energy difference between them. Note that these adiabatic states are the eigenstates of the Hamiltonian at fixed time $t$.

Under a time-dependent interaction, i. e., in our case the interaction with the laser field $H_{\mathrm{L}}(t)(4)$, the dynamics could be considered as adiabatic if and only if the following condition 35.

$$
\eta=\frac{\left.\hbar\right|_{\mathrm{p}}\left\langle i\left|\frac{\partial H_{\mathrm{L}}(t)}{\partial t}\right| j\right\rangle_{\mathrm{p}} \mid}{\left|E_{i}-E_{j}\right|^{2}} \ll 1
$$

is fulfilled, with $|i\rangle_{\mathrm{p}}$ and $|j\rangle_{\mathrm{p}}$ being the eigenstates of the Hamiltonian in the adiabatic limit, and $E_{i}$ and $E_{j}$ their energies. According to this criterion, the probability for mixing, corresponding to a transfer of population from one state of the doublet to the other, is determined by the rate of change of the laser field interaction and the energy separation between the states. Thus, as the laser intensity is increased the population transfer between the two states in a doublet takes place because the criterion (6) is not satisfied. To illustrate this phenomenon, we show the contribution of the adiabatic ground state $\left|C_{00 e}(t)\right|^{2}$ to the time-dependent wave function of $|0,0, e\rangle^{0}$, Fig. 3 (b), and the adiabatic parameter $\eta$ when $\eta \geq 10^{-4}$, Fig. 3. (c). Note that $\left|C_{00 e}(t)\right|^{2}+\left|C_{10 e}(t)\right|^{2}=1$. In these four cases, the dynamics is initially adiabatic, i. e., $\left|C_{00 e}(t)\right|^{2}$ remains equal to 1 and $\eta \ll 1$. As $\mathrm{I}(t)$ is increased, the energy splitting of the doublet decreases and, moreover, it becomes comparable or even larger than the rate of turningon the pulse; thus, $\eta$ is close to 1 , and the population transfer takes place. This region where $\eta$ is not negligible corresponds to the formation of the quasi-degenerate doublet. Once the doublet is formed, $\Delta E=\left|E_{i}-E_{j}\right|$ reaches a small value and slowly decreases as $\mathrm{I}(t)$ is enhanced; but the two states are oriented in opposite directions and their wave functions do not overlap. Therefore, the coupling due to the alignment laser is much smaller than $\Delta E, \eta \ll 1$ and the population transfer does not take place any longer, i.e., $\left|C_{00 e}(t)\right|^{2}$ remains constant as $\mathrm{I}(t)$ is enhanced. The larger is this population transfer, the smaller is the orientation compared to the adiabatic prediction. Since these adiabatic states contributing to the dynamics are quasi-degenerated and have very close values of the alignment and hybridization of the angular motion, the lack of adiabaticity is not reflected on the time evolution of the energy, $\left\langle\cos ^{2} \theta\right\rangle$ or $\left\langle\mathbf{J}^{2}\right\rangle$.

For this field configuration, the molecular dynamics of excited states present analogous features for $\langle\cos \theta\rangle$, $\left\langle\cos ^{2} \theta\right\rangle$ and $\left\langle\mathbf{J}^{2}\right\rangle$ as those encountered here for the ground state.

The adiabaticity of the field-dressed dynamics is determined by the rate of change of the laser field interaction compared to the largest time scale of the system. In the pendular regime, the energy splitting in a doublet goes as $\Delta E \sim 2 \mu \mathrm{E}_{\mathrm{s}}$; then, the population transfer decreases if $\mathrm{E}_{\mathrm{s}}$ is increased. On the other hand, by increasing the FWHM augments the time scale on which the pendu- 
lar doublets are formed, and facilitates the adaptation of the molecule to this field. That is, the mixed-field orientation will be more adiabatic when either longer pulses or stronger static electric fields are used. Let us remark that with the expression being the dynamics more adiabatic we mean that for a certain state, the weight of its corresponding adiabatic state in the time-dependent wave function is closer to one during the dynamics.

\section{A. Influence of the peak intensity $\mathbf{I}_{0}$}

Here, we investigate the orientation at the maximum of the laser pulse, as it is done in most of the experiments [20, 24, 36]. The rate of change of the laser field and the adiabatic parameter (6) depend linearly on $\mathrm{I}_{0}$. Then, for a Gaussian pulse with fixed FWHM, the dynamics will be more diabatic if $I_{0}$ is increased. In this section, we consider the states $|0,0, e\rangle^{0},|1,0, e\rangle^{0},|1,1, e\rangle^{0},|2,1, e\rangle^{0}$, $|2,0, e\rangle^{0},|3,0, e\rangle^{0},|2,2, e\rangle^{0}$, and $|3,2, e\rangle^{0}$. Their orientation at $t=0$, i. e., $\langle\cos \theta\rangle$ for $\mathrm{I}(0)=\mathrm{I}_{0}$, is plotted as a function of $\mathrm{I}_{0}$ in Fig. 4 (a) and (b) for $\mathrm{E}_{\mathrm{s}}=300 \mathrm{~V} / \mathrm{cm}$ and $600 \mathrm{~V} / \mathrm{cm}$, respectively. The fields are parallel, and the FWHM of these pulses is $10 \mathrm{~ns}$.

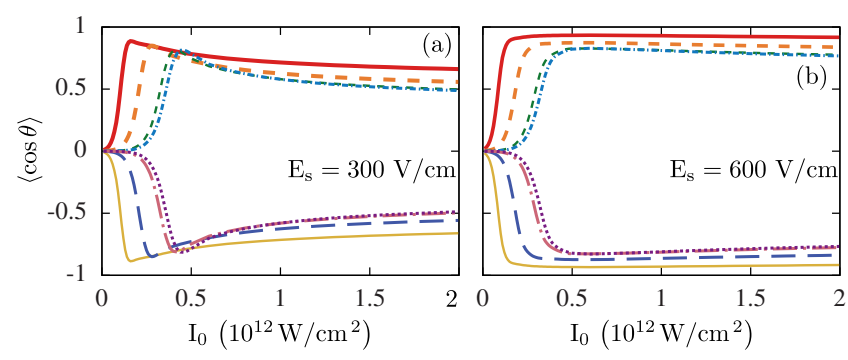

FIG. 4. (Color online) Expectation value $\langle\cos \theta\rangle$ at $t=0$ as a function of the peak intensity $\mathrm{I}_{0}$ for the states $|0,0, e\rangle^{0}$ (red thick solid), $|1,0, e\rangle^{0}$ (gold thin solid) $|1,1, e\rangle^{0}$ (orange thick short-dashed), $|2,1, e\rangle^{0}$ (dark blue long-dashed) $|2,0, e\rangle^{0}$ (blue dot-short-dashed), $|3,0, e\rangle^{0}$ (purple dotted), $|2,2, e\rangle^{0}$ (green thin short-dashed) and $|3,2, e\rangle^{0}$ (pink dot-long-dashed), for $\beta=0^{\circ}$ and (a) $\mathrm{E}_{\mathrm{s}}=300 \mathrm{~V} / \mathrm{cm}$ and (b) $600 \mathrm{~V} / \mathrm{cm}$. The FWHM of the laser pulses is $10 \mathrm{~ns}$.

For $\mathrm{E}_{\mathrm{s}}=300 \mathrm{~V} / \mathrm{cm}$, the orientation of the low-lying level in a doublet increases as $I_{0}$ is enhanced, reaching a maximum and smoothly decreasing thereafter. This is counterintuitive to what is expected in the adiabatic limit; namely, a larger orientation when the laser intensity is increased. The maximum in the orientation is achieved with an alignment pulse that already gives rise to a non-adiabatic dynamics. However, due to the coupling between the populated adiabatic states in the pendular pair ${ }_{\mathrm{p}}\langle i|\cos \theta| j\rangle_{\mathrm{p}}$, the orientation is enhanced compared to what happens at the adiabatic limit. By further increasing $\mathrm{I}_{0}$, the population transferred between the two states is enhanced, but now the coupling between them is very small or even zero due to their opposite orientation. As a consequence, the final orientation decreases as
$\mathrm{I}_{0}$ is increased. For a certain pendular doublet, the upper state is antioriented, and $\langle\cos \theta\rangle$ shows the opposite behavior as a function of $\mathrm{I}_{0}$. Regarding the third and fourth doublets, the states are not oriented nor aligned for ac pulses with $\mathrm{I}_{0} \lesssim 2.4 \times 10^{11} \mathrm{~W} / \mathrm{cm}^{2}$. Compared to low-lying states, their orientation is smaller and the maximum of $\langle\cos \theta\rangle$ appears at larger peak intensities.

By increasing the static field strength to $600 \mathrm{~V} / \mathrm{cm}$ the energy gap of the pendular pair is also increased, being the characteristic time scale of the system reduced. Thus, for the same laser pulse, the dynamics is more adiabatic, i. e., less population is transferred, and the final orientation is increased, see Fig. 4(b). The orientation (antiorientation) of the pendular states also achieves a maximum (minimum), but it is so shallow that it is hardly appreciated on the scale of this figure.

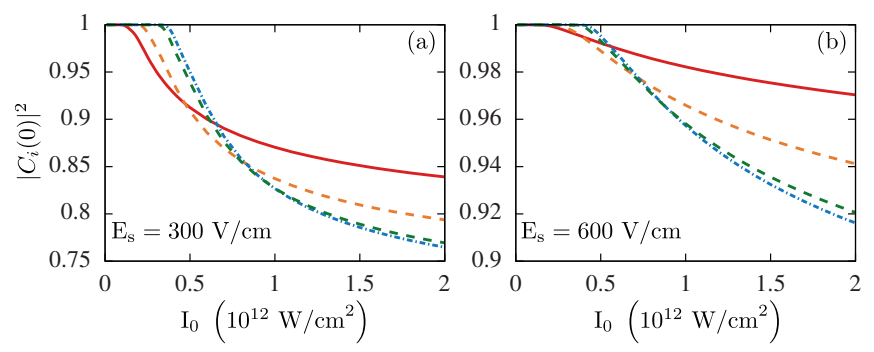

FIG. 5. (Color online) Projections of the time-dependent wave functions onto the corresponding adiabatic states as a function of the peak intensity $\mathrm{I}_{0}$ for the states $|0,0, e\rangle^{0}$ (red thick solid), $|1,1, e\rangle^{0}$ (orange thick short-dashed), $|2,0, e\rangle^{0}$ (blue dot-short-dashed) and $|2,2, e\rangle^{0}$ (green thin shortdashed), for (a) $\mathrm{E}_{\mathrm{s}}=300 \mathrm{~V} / \mathrm{cm}$ and (b) $600 \mathrm{~V} / \mathrm{cm}$. We use 10 ns laser pulses and $\beta=0^{\circ}$.

To illustrate the field-dressed dynamics, we plot in Fig. 5 the weights of the adiabatic states associated to the oriented levels in these pendular doublets. For the corresponding antioriented levels, the contributions of its associated adiabatic state are identical to the one presented here, e.g., for the ground state, we present the contribution of the adiabatic ground state $\left|C_{00 e}(0)\right|^{2}$, which is identical to the weight $\left|C_{10 e}(0)\right|^{2}$ for $|1,0, e\rangle$. In an adiabatic molecular dynamics, these coefficients are equal to one. Note that in the considered regime only the two adiabatic states of the pendular doublet contribute to the dynamics. For all these levels, $\left|C_{i}(0)\right|^{2}$ decreases, i. e., the dynamics is less adiabatic, as $\mathrm{I}_{0}$ is enhanced. By increasing $\mathrm{E}_{\mathrm{s}}, \Delta E$ is increased; thus, under the same Gaussian pulse the population transfer is reduced, i.e., $\left|C_{i}(0)\right|^{2}$ is closer to one, and the range of peak intensities that could be considered as adiabatic is increased.

\section{B. Influence of the FWHM of the laser pulse}

The duration of the Gaussian pulse plays an important role in the molecular dynamics. It has been shown that an alignment pulse of $10 \mathrm{~ns}$ is not enough to achieve 
an adiabatic mixed-field orientation for molecules such as OCS, benzonitrile and iodobenzene [19, 26, 34, 37]. It has been pointed out the need of increasing the rising time of the laser pulses to achieve the highest possible orientation 26, 38, 39]. By increasing the FWHM, the time needed to form the pendular doublets is also increased. For a given field configuration, at the point where the pulse reaches a certain intensity the adiabatic parameter (6) is reduced if $\tau$ is increased. Hence, the molecular dynamics becomes more adiabatic and, therefore, the population transfer to other pendular states is reduced. Here, we investigate how the directional properties of OCS depends on the laser-pulse FWHM. For the same set of states as in the previous section, Fig. 6 shows the orientation at $t=0$ as a function of $\tau$. The fields are parallel, and we consider the peak intensities $\mathrm{I}_{0}=2 \times 10^{11} \mathrm{~W} / \mathrm{cm}^{2}$ and $10^{12} \mathrm{~W} / \mathrm{cm}^{2}$, and a dc field of $\mathrm{E}_{\mathrm{s}}=300 \mathrm{~V} / \mathrm{cm}$.
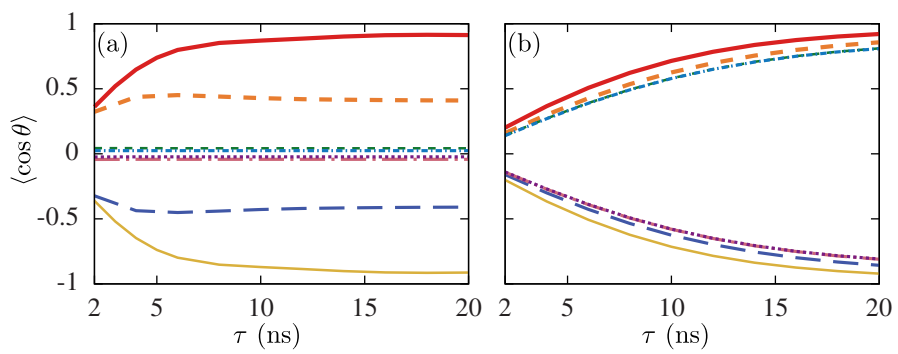

FIG. 6. (Color online) Expectation value $\langle\cos \theta\rangle$ at $t=0$ as a function of $\tau$ for (a) $\mathrm{I}_{0}=2 \times 10^{11} \mathrm{~W} / \mathrm{cm}^{2}$ and (b) $\mathrm{I}_{0}=$ $10^{12} \mathrm{~W} / \mathrm{cm}^{2}$. The fields are parallel and $\mathrm{E}_{\mathrm{s}}=300 \mathrm{~V} / \mathrm{cm}$. The states and their labels are the same as in Fig. 4

The degree of orientation of the two states in a given pendular pair shows the same behavior as a function of $\tau$, but with their dipole moment pointing in opposite directions. In the first two doublets and $\mathrm{I}_{0}=2 \times 10^{11} \mathrm{~W} / \mathrm{cm}^{2}$, $|\langle\cos \theta\rangle|$ increases with $\tau$ till it reaches a plateau-like behavior. The second pair satisfies that $|\langle\cos \theta\rangle| \approx 0.428$ for $\tau \gtrsim 10 \mathrm{~ns}$. Since the states in the third and fourth doublets have not achieved the pendular regime for $\mathrm{I}_{0}=2 \times 10^{11} \mathrm{~W} / \mathrm{cm}^{2}$, increasing the pulse duration does not have any impact on their orientation, and $\langle\cos \theta\rangle$ keeps a constant value close to zero as $\tau$ is increased. For $\mathrm{I}_{0}=10^{12} \mathrm{~W} / \mathrm{cm}^{2}$, the degree of orientation of all the states increases and approaches the adiabatic limit as $\tau$ is enhanced. For the ground state and $\tau=20 \mathrm{ns,} \mathrm{we} \mathrm{ob-}$ tain $\langle\cos \theta\rangle=0.913$, which is very close to the adiabatic value $\langle\cos \theta\rangle=0.964$.

These results show that for parallel fields, the mixedfield orientation dynamics of any state could be adiabatic if a sufficiently long pulse and sufficiently strong fields are used. For a $50 \mathrm{~ns}$ Gaussian pulse with $\mathrm{I}_{0}=10^{12} \mathrm{~W} / \mathrm{cm}^{2}$, and $\mathrm{E}_{\mathrm{s}}=300 \mathrm{~V} / \mathrm{cm}$, the dynamics can be considered as adiabatic for all these states, with $\left|C_{i}(0)\right|^{2} \gtrsim 0.999$.

\section{Influence of the electrostatic field strength}

Since the energy splitting in a pendular doublet is proportional to the static field strength, the degree of adiabaticity in the molecular orientation should increase if $\mathrm{E}_{\mathrm{S}}$ is enhanced, i. e., the characteristic time scale of the system is reduced. In Fig. 7 we present the final orientation at $t=0$ of these eight states versus $\mathrm{E}_{\mathrm{s}}$. We have considered two laser pulses of $\tau=10 \mathrm{~ns}$ with peak intensities $\mathrm{I}_{0}=2 \times 10^{11} \mathrm{~W} / \mathrm{cm}^{2}$ and $10^{12} \mathrm{~W} / \mathrm{cm}^{2}$, and $\beta=0^{\circ}$.
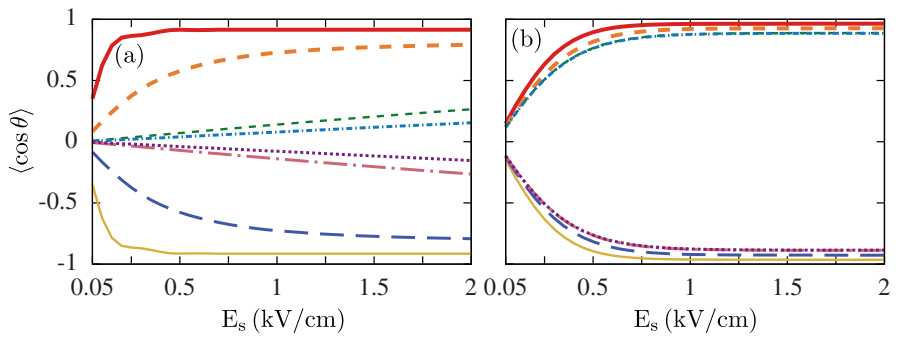

FIG. 7. (Color online) Expectation value $\langle\cos \theta\rangle$ at $t=0$ as a function of $\mathrm{E}_{\mathrm{s}}$ for (a) $\mathrm{I}_{0}=2 \times 10^{11} \mathrm{~W} / \mathrm{cm}^{2}$ and (b) $\mathrm{I}_{0}=$ $10^{12} \mathrm{~W} / \mathrm{cm}^{2}$. We use a $10 \mathrm{~ns}$ laser pulses and $\beta=0^{\circ}$. The states and their labels are the same as in Fig. 4.

For the lowest laser intensity, the orientation of the $|0,0, e\rangle^{0}$ and $|1,0, e\rangle^{0}$ states is constant and independent of the static field for $\mathrm{E}_{\mathrm{s}} \gtrsim 500 \mathrm{~V} / \mathrm{cm}$ with $|\langle\cos \theta\rangle|=$ 0.915. The orientation of the levels $|1,1, e\rangle^{0}$ and $|2,1, e\rangle^{0}$ monotonically increases as $\mathrm{E}_{\mathrm{s}}$ is enhanced, and we obtain $|\langle\cos \theta\rangle|=0.792$ for $\mathrm{E}_{\mathrm{s}}=2 \mathrm{kV} / \mathrm{cm}$. This peak intensity is not large enough for the states in the third and fourth doublets to be in the pendular regime. Thus, these pairs are weakly oriented even if a strong dc field is used, e.g., for $\mathrm{E}_{\mathrm{s}}=2 \mathrm{kV} / \mathrm{cm},|\langle\cos \theta\rangle|=0.265$ and 0.153 for the third and fourth doublets, respectively.

For the strong peak intensity, all the states are in the pendular regime, and their $|\langle\cos \theta\rangle|$ increases as $\mathrm{E}_{\mathrm{s}}$ is increased reaching a constant value for sufficiently strong static fields. Their orientation approaches the adiabatic limit and for $\mathrm{E}_{\mathrm{s}} \gtrsim 1 \mathrm{kV} / \mathrm{cm},|\langle\cos \theta\rangle|=0.949$ for the states of the first doublet, and $99.91 \%$ of their population is on the corresponding adiabatic pendular state. For $\mathrm{E}_{\mathrm{s}}=2 \mathrm{kV} / \mathrm{cm}$, the states in the fourth doublet satisfy $|\langle\cos \theta\rangle|=0.885$ and $99.99 \%$ of their population is on the corresponding adiabatic level.

In conclusion, by combining sufficiently strong electrostatic fields with standard Gaussian pulses, i. e., with experimentally accessible peak intensities of $10^{12} \mathrm{~W} / \mathrm{cm}^{2}$ and 10 ns FWHM, a significant orientation is obtained even for excited rotational levels. It is worth remarking that the fields have to be parallel; then, techniques such us the ion imaging method [24] could not be used to measure the degree of orientation; whereas techniques, such as time of flight 20, 36] are feasible. 


\section{RESULTS FOR NON-PARALLEL FIELDS}

In this section we investigate the rotational dynamics when the electrostatic field forms an angle $0^{\circ}<\beta<90^{\circ}$ with the linearly polarized laser pulse. The azimuthal symmetry is lost, and the number of irreducible representations is reduced to two, see Sec. II]. Thus, states with different field-free magnetic quantum numbers are now coupled by the electrostatic field.

The field-free wave function of the initial state is constructed as an eigenstate of the operators $\mathcal{C}_{\text {Es }}(\pi)$ and $\sigma_{X Z}$, see Table I; i. e., $|J, M, e\rangle=R_{Y}(\beta)|J, M, e\rangle^{0}$, where $R_{Y}(\beta)$ is the rotation operator of an angle $\beta$ around the LFF $Y$-axis 40 ]. This ensures that this wave function has the correct symmetries, and that its time evolution corresponds, in the adiabatic limit, to an eigenstate of the field-dressed Hamiltonian at any time.

Before the pulse is turned on, an important feature of the ground state is that its energy gap to the next state with the same symmetry is proportional to the rotational constant $B$, which is much larger than the coupling in the weak laser field regime. In this regime, it evolves as an isolated state, and its interaction to the neighboring levels could be considered negligible. Hence, analogously to the parallel field configuration, the formation of the doublets in the pendular regime is the only source of nonadiabatic effects in its field-dressed dynamics. Note that the lowest lying level of the odd irreducible representation will show the same behavior.
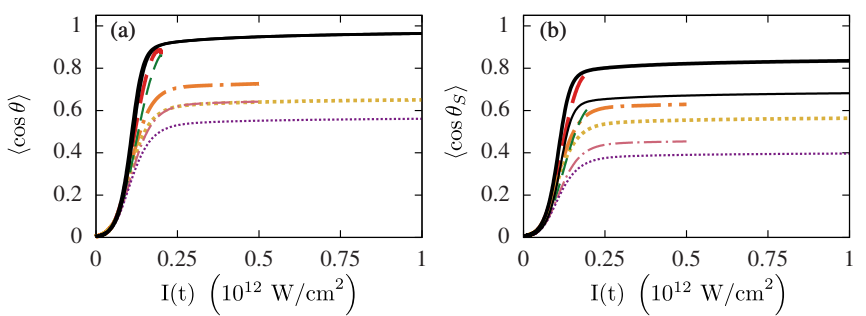

FIG. 8. (Color online) For the ground state, evolution of the expectation values (a) $\langle\cos \theta\rangle$ and (b) $\left\langle\cos \theta_{\mathrm{s}}\right\rangle$ as a function of $\mathrm{I}(t)$ of a $10 \mathrm{~ns}$ Gaussian pulse. The field configurations are $\beta=30^{\circ}$ (thick) and $45^{\circ}$ (thin) with peak intensities $\mathrm{I}_{0}=$ $2 \times 10^{11} \mathrm{~W} / \mathrm{cm}^{2}$ (dashed), $5 \times 10^{11} \mathrm{~W} / \mathrm{cm}^{2}$ (dot-dashed), and $10^{12} \mathrm{~W} / \mathrm{cm}^{2}$ (dotted). The static electric field is fixed to $\mathrm{E}_{\mathrm{s}}=$ $300 \mathrm{~V} / \mathrm{cm}$. The adiabatic results (solid) are also included.

For the ground state, the time evolution of the expectation values $\langle\cos \theta\rangle$ and $\left\langle\cos \theta_{\mathrm{s}}\right\rangle$ are presented as a function of $\mathrm{I}(t)$ till the peak intensity is reached in Fig. 8 (a) and (b). The Gaussian pulse has 10 ns FWHM and peak intensities $\mathrm{I}_{0}=2 \times 10^{11} \mathrm{~W} / \mathrm{cm}^{2}, 5 \times 10^{11} \mathrm{~W} / \mathrm{cm}^{2}$, and $10^{12} \mathrm{~W} / \mathrm{cm}^{2}$. We consider the inclination angles $\beta=30^{\circ}$ and $45^{\circ}$, and a dc field of $\mathrm{E}_{\mathrm{s}}=300 \mathrm{~V} / \mathrm{cm}$. For comparison, the adiabatic results are also included, with $\langle\cos \theta\rangle$ being independent of $\beta$. For a certain laser pulse, increasing the inclination angle towards $90^{\circ} \mathrm{im}-$ plies a decrease of the energy splitting in the first doublet, $\Delta E \sim 2 \mu \mathrm{E}_{\mathrm{s}} \cos \beta$, and, therefore, an increase of the adiabatic parameter (6). Note that in the energy splitting we have not considered the component of the dc field along the LFF $X$-axis. Compared to the $\beta=0^{\circ}$ configuration, the dynamics could be considered as less adiabatic being characterized by a larger population transfer to the other adiabatic state in this pendular pair. The final orientation is significantly decreased as $\beta$ is increased, e.g., for $\mathrm{I}_{0}=10^{12} \mathrm{~W} / \mathrm{cm}^{2},\langle\cos \theta\rangle=0.651$ and 0.561 , and the contribution of the adiabatic ground state is $\left|C_{00 e}(0)\right|^{2}=0.837$ and 0.791 for $\beta=30^{\circ}$ and $45^{\circ}$, respectively. For a certain angle $\beta$, the orientation achieved at $t=0$ decreases as $\mathrm{I}_{0}$ is increased, cf. Fig. 8. Since the molecular dynamics of the ground state is restricted to the two lower pendular adiabatic states, its time dependent results for its energy and expectation values $\left\langle\cos ^{2} \theta\right\rangle$ and $\left\langle\mathbf{J}^{2}\right\rangle$ resemble the adiabatic ones.

To illustrate the rotational dynamics of excited states, we show in Fig. 9 (a) the orientation cosine $\langle\cos \theta\rangle$ as a function of $\mathrm{I}(t)$ for $|1,1, e\rangle$. The field configurations are the same as in Fig. 8. The adiabatic model predicts a sharp wrong-way orientation. In contrast, this state presents a weak or even zero orientation, and in addition, a larger peak intensity does not imply a larger orientation. When the peak intensity is reached, this level shows a weak right-way orientation for $\beta=30^{\circ}$ : $\langle\cos \theta\rangle=0.326$ and 0.259 , for $\mathrm{I}_{0}=5 \times 10^{11} \mathrm{~W} / \mathrm{cm}^{2}$ and $10^{12} \mathrm{~W} / \mathrm{cm}^{2}$, respectively. For $\beta=45^{\circ}$ and the peak intensities $\mathrm{I}_{0}=5 \times 10^{11} \mathrm{~W} / \mathrm{cm}^{2}$ and $10^{12} \mathrm{~W} / \mathrm{cm}^{2}$, due to the non-adiabatic dynamics $|1,1, e\rangle$ is not oriented.

Let us analyze in detail these results. For highly excited states, the dynamics is more complicated. Apart from the doublet formation, there is another physical phenomenon at weak laser intensities which causes loss of adiabaticity. In the presence of only a weak static field, the $M$-degeneracy of the states with the same field-free $J$ is broken due to the quadratic Stark effect, i. e., the splitting goes as $\Delta E \sim \mathrm{E}_{\mathrm{s}}^{2}$. As the pulse is switched on, the energy gap between two states of this $J$-manifold is much smaller than the rate of their coupling due to the laser field, i. e., $\eta$ is larger than one. For $\beta=30^{\circ}$, the adiabatic parameter $\eta$ between the states $|1,1, e\rangle_{\mathrm{p}}$ and $|1,0, e\rangle_{\mathrm{p}}$, both contributing to the dynamics of $|1,1, e\rangle$, is presented in Fig. 9 (b), and it achieves large values for $\mathrm{I}(t) \lesssim 5 \times 10^{5} \mathrm{~W} / \mathrm{cm}^{2}$. As the states in this $J$-manifold are driven apart by the laser field, the process is non-adiabatic and there is a population transfer between them. The projections of the time-dependent wave function in terms of the adiabatic states $|0,0, e\rangle_{\mathrm{p}},|1,1, e\rangle_{\mathrm{p}},|1,0, e\rangle_{\mathrm{p}}$, and $|2,2, e\rangle_{\mathrm{p}}$ is presented in Fig. 9 (d) for $\beta=30^{\circ}$ and $\mathrm{I}_{0}=10^{12} \mathrm{~W} / \mathrm{cm}^{2}$. Under these diabatic conditions, $\left|C_{11 e}(t)\right|^{2}$ decreases as $\mathrm{I}(t)$ is increased, whereas $\left|C_{10 e}(t)\right|^{2}$ increases. By further increasing $\mathrm{I}(t)$, the coupling between these states becomes very small or even zero and their energy separation increases, so that $\eta$ decreases and the population transfer is stopped. This process is so diabatic that the wave function does not change, but its projections on the adiabatic basis are modified because the basis 
varies with time. For instance, the field-free state is $|1,1, e\rangle=\cos \beta|1,1, e\rangle_{\mathrm{p}}^{0}+\sin \beta|1,0, e\rangle_{\mathrm{p}}^{0}$, which belongs to the proper irreducible representation. After swichting on the static field, its wave function could be approximated by the same expression because this field is very weak. Once the splitting of this $J$-manifold is finished, i. e., for $\mathrm{I}(t) \sim 5 \times 10^{7} \mathrm{~W} / \mathrm{cm}^{2}$, the contributions of the states $|1,1, e\rangle_{\mathrm{p}}$ and $|1,0, e\rangle_{\mathrm{p}}$ are approximately $\sin ^{2} \beta$ and $\cos ^{2} \beta$, respectively. Note that the states $|J, M, e\rangle_{\mathrm{p}}^{0}$ and $|J, M, e\rangle_{\mathrm{p}}$ are not related adiabatically.
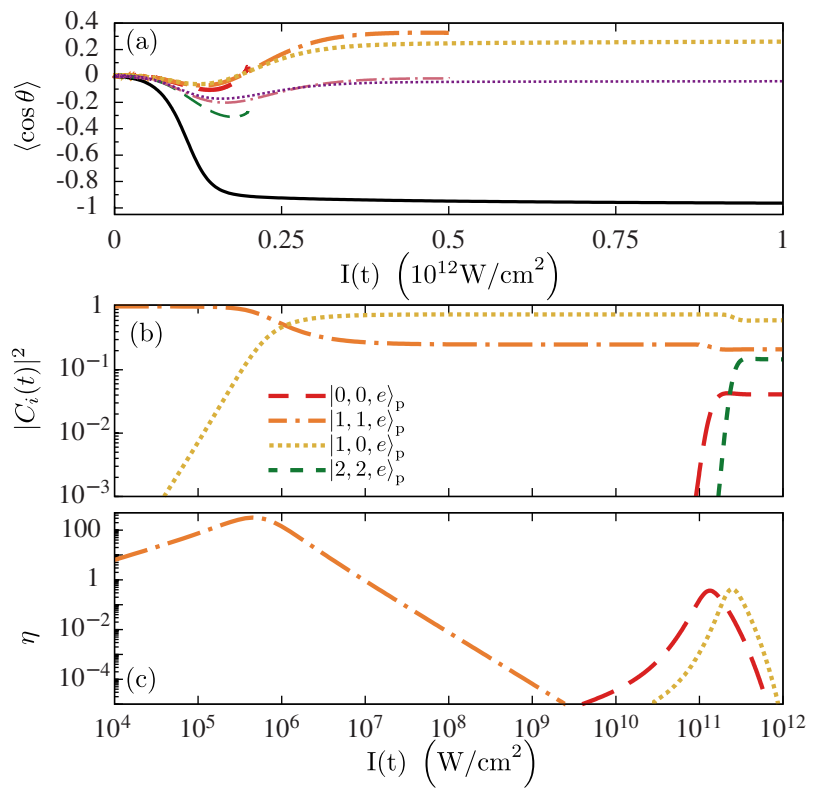

FIG. 9. (Color online) (a) For the $|1,1, e\rangle$ state, expectation value $\langle\cos \theta\rangle$ as a function of $\mathrm{I}(t)$ of a 10 ns Gaussian pulse. The field configurations are $\beta=30^{\circ}$ (thick) and $45^{\circ}$ (thin), with $\mathrm{I}_{0}=2 \times 10^{11} \mathrm{~W} / \mathrm{cm}^{2}$ (dashed), $5 \times 10^{11} \mathrm{~W} / \mathrm{cm}^{2}$ (dot-dashed) and $10^{12} \mathrm{~W} / \mathrm{cm}^{2}$ (dotted). The adiabatic results (solid line) are also included. For $\mathrm{I}_{0}=10^{12} \mathrm{~W} / \mathrm{cm}^{2}$ and $\beta=30^{\circ}$, (b) square of the projections of the time-dependent wave function on the adiabatic pendular states $|1,1, e\rangle_{\mathrm{p}}$ (dot-dashed), $|1,0, e\rangle_{\mathrm{p}}$ (dotted), $|0,0, e\rangle_{\mathrm{p}}$ (long-dashed), and $|2,2, e\rangle_{\mathrm{p}}$ (short-dashed), and (c) adiabatic parameter between the pendular states $|1,1, e\rangle_{\mathrm{p}}$ and $|1,0, e\rangle_{\mathrm{p}}$ (dot-dashed), $|1,1, e\rangle_{\mathrm{p}}$ and $|0,0, e\rangle_{\mathrm{p}}$ (dashed), and $|1,0, e\rangle_{\mathrm{p}}$ and $|2,2, e\rangle_{\mathrm{p}}$ (dotted). The dc field is fixed to $\mathrm{E}_{\mathrm{s}}=300 \mathrm{~V} / \mathrm{cm}$.

In contrast to the ground state, the wave function of any excited level has contributions from adiabatic states which correspond to different pendular doublets. As the laser intensity is increased, the molecular dynamics is affected by the formation of these pendular doublets. Thus, the final orientation could be significantly reduced compared to the parallel fields result. For instance, the timedependent $|1,1, e\rangle$ state has contributions from the adiabatic levels $|1,1, e\rangle_{\mathrm{p}}$ and $|1,0, e\rangle_{\mathrm{p}}$, which correspond to the first and second pendular doublets, respectively. In Fig. 9 (b) we show how the adiabatic parameters $\eta$ between the pairs $|0,0, e\rangle_{\mathrm{p}}-|1,1, e\rangle_{\mathrm{p}}$ and $|1,0, e\rangle_{\mathrm{p}}-|2,2, e\rangle_{\mathrm{p}}$, which form the first and second doublets, respectively, achieve values close to 1 . The final population of the state $|1,1, e\rangle$ is $\left|C_{00 e}(0)\right|^{2}=0.041,\left|C_{11 e}(0)\right|^{2}=0.210$, $\left|C_{10 e}(0)\right|^{2}=0.603$, and $\left|C_{22 e}(0)\right|^{2}=0.146$, which gives rise to a small orientation As a consequence of this population redistribution to other pendular doublets, features of the system such as energy, alignment and hybridization of the angular motion do not resemble the adiabatic results. In particular, since the levels on the second pendular doublet possess a smaller alignment, the adiabatic result is larger than the time-dependent one. For instance, for $\beta=30^{\circ}$ and $\mathrm{I}_{0}=10^{12} \mathrm{~W} / \mathrm{cm}^{2}$, once the time evolution is finished the alignment of this state $|1,1, e\rangle$ is $\left\langle\cos ^{2} \theta\right\rangle=0.879$, compared to $\left\langle\cos ^{2} \theta\right\rangle=0.931$ obtained for the adiabatic level $|1,1, e\rangle_{\mathrm{p}}$.

For $\beta=45^{\circ}$, despite the fact that the $|1,1, e\rangle$ level is significantly aligned, $\left\langle\cos ^{2} \theta\right\rangle=0.896$, it is not oriented with $\langle\cos \theta\rangle=-0.041$ for $\mathrm{I}_{0}=10^{12} \mathrm{~W} / \mathrm{cm}^{2}$. This state does not gain any orientation if stronger peak intensities are used. This is a consequence of the population redistribution explained above. Indeed, this level could be considered as a dark state for the mixed-field orientation dynamics. This physical phenomenon is not restricted to this state and field configuration. We show below that other levels also behave as dark states. It is worth noting that if in a mixed-field orientation experiment these dark states form part of the molecular beam, the degree of orientation will be diminished.

The population redistribution to other pendular doublets significantly affects the expectation value $\left\langle\cos \theta_{\mathrm{s}}\right\rangle$. To $\left\langle\cos \theta_{\mathrm{s}}\right\rangle$ contribute terms which mix up adiabatic states with different magnetic quantum numbers Since their wave functions could spatially overlap, their coupling matrix elements do not vanish, and $\left\langle\cos \theta_{\mathrm{s}}\right\rangle$ oscillates as $t$ is increased.

The phenomenon of population redistribution at weak laser intensities also occurs for highly excited rotational levels, and for them, more adiabatic states would be involved in it. Before the Gaussian pulse is turned on, the Stark separation of the states in a certain $J$-manifold is increased if the electrostatic field strength is enhanced. Then, the adiabatic parameter $\eta$ is reduced, and the process of splitting of this $J$-manifold becomes less diabatic. Indeed, for sufficiently strong dc-fields, the dynamics would be adiabatic without population transfer between the states with the same field-free $J$. For instance, the mixed-field dynamics of the $|1,1, e\rangle$ level can be considered as adiabatic on the weak laser field regime for $\mathrm{E}_{\mathrm{s}} \gtrsim 14 \mathrm{kV} / \mathrm{cm}$ and $\beta=30^{\circ}$.

Let us remark that the excited states could also suffer avoided crossings with adjacent levels, having different field-free magnetic quantum number $M$, as the pulse intensity is varied. The rotational dynamics in most of these crossings will be non-adiabatic 25]. 


\section{A. Influence of the peak intensity $\mathbf{I}_{0}$}

Analogously to the parallel-field configuration, we investigate now the impact of the laser peak intensity on the orientation. To do so, we restrict this study to the following eight states: $|0,0, e\rangle,|1,0, e\rangle,|1,1, e\rangle,|2,0, e\rangle$, $|2,1, e\rangle,|2,2, e\rangle,|3,0, e\rangle$, and $|3,2, e\rangle$. Note that they are related to the ones analyzed in the parallel fields configuration, by a rotation of $\beta$ around the LFF $Y$ axis. Their orientation at $t=0$, i. e., $\langle\cos \theta\rangle$ for $\mathrm{I}(0)=\mathrm{I}_{0}$, is plotted as a function of $\mathrm{I}_{0}$ in Fig. 10 for $\beta=30^{\circ}$, panels (a)-(b), $\beta=45^{\circ}$, panels (c)-(d), and $\beta=75^{\circ}$, panels (e)-(f), and $\mathrm{E}_{\mathrm{s}}=300 \mathrm{~V} / \mathrm{cm}$ and $600 \mathrm{~V} / \mathrm{cm}$, respectively. The FWHM of these Gaussian pulses is fixed to $\tau=10 \mathrm{~ns}$.
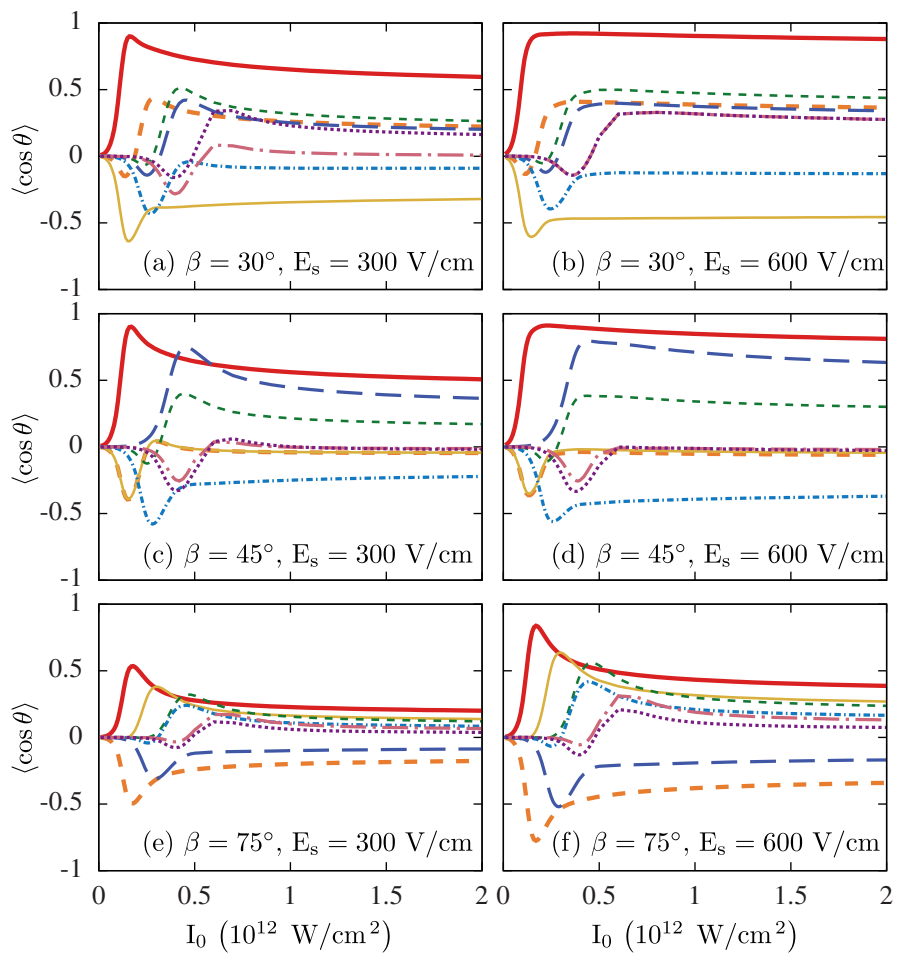

FIG. 10. (Color online) Expectation value $\langle\cos \theta\rangle$ at $t=0$ as a function of the peak intensity $\mathrm{I}_{0}$ for $|0,0, e\rangle$ (red thick solid), $|1,0, e\rangle$ (gold thin solid) $|1,1, e\rangle$ (orange thick shortdashed), $|2,1, e\rangle$ (dark blue long-dashed) $|2,0, e\rangle$ (blue dotshort-dashed), $|3,0, e\rangle$ (purple dotted), $|2,2, e\rangle$ (green thin short-dashed) and $|3,2, e\rangle$ (pink dot-long-dashed). The field configurations are (a)-(b) $\beta=30^{\circ}$, (c)-(d) $\beta=45^{\circ}$ and (e)(f) $\beta=75^{\circ}$, with $\mathrm{E}_{\mathrm{s}}=300 \mathrm{~V} / \mathrm{cm}$ and $600 \mathrm{~V} / \mathrm{cm}$, respectively. The FWHMs of the Gaussian pulses are fixed to $10 \mathrm{~ns}$.

Let us start analyzing the results for the ground state. For all field configurations, $\langle\cos \theta\rangle$ shows a qualitatively similar behavior as a function of the peak intensity: initially increases, reaches a maximum and decreases thereafter. At the peak intensity where the maximum of $\langle\cos \theta\rangle$ takes place, the dynamics of this state is nonadiabatic, but due to the coupling of both states the orientation increases with respect to the adiabatic result. For a fixed peak intensity and electric field strength, $\langle\cos \theta\rangle$ decreases as $\beta$ is increased towards $90^{\circ}$. For $\beta=75^{\circ}$ and $\mathrm{I}_{0}=2 \times 10^{11} \mathrm{~W} / \mathrm{cm}^{2}$, the ground state achieves a moderate maximal orientation, $\langle\cos \theta\rangle=0.514$ and 0.796 for $\mathrm{E}_{\mathrm{s}}=300 \mathrm{~V} / \mathrm{cm}$ and $600 \mathrm{~V} / \mathrm{cm}$, respectively.

The population transfer taking place at weak and strong laser intensities leaves its finger-prints in the dynamics of the excited states. Compared to the parallel field results, cf Fig. 4 their orientation is reduced for any inclination angle $\beta$ and the pendular pairs are not any longer formed by a right- and wrong-way oriented states. Whereas for most of the field configurations, the ground state possesses the largest orientation, the levels $|1,0, e\rangle$ or $|2,1,0\rangle$ could achieve a similar or even larger orientation, e.g., for $\beta=45^{\circ}$ and $75^{\circ}, \mathrm{I}_{0} \approx 5 \times 10^{11} \mathrm{~W} / \mathrm{cm}^{2}$ and $\mathrm{E}_{\mathrm{s}}=300 \mathrm{~V} / \mathrm{cm}$. For $\beta=30^{\circ} \mathrm{E}_{\mathrm{s}}=600 \mathrm{~V} / \mathrm{cm}$, the degree of orientation is moderate for most of the states. Several dark states are found for $\beta=45^{\circ}:|1,1, e\rangle,|1,0, e\rangle,|3,0, e\rangle$, and $|3,2, e\rangle$, see Fig. 4 (c)-(d). For instance, the levels $|1,1, e\rangle$ and $|1,0, e\rangle$ are strongly aligned with $\left\langle\cos ^{2} \theta\right\rangle=0.927$ for $\mathrm{I}_{0} \gtrsim 2 \times 10^{12} \mathrm{~W} / \mathrm{cm}^{2}$ and $\mathrm{E}_{\mathrm{s}}=600 \mathrm{~V} / \mathrm{cm}$, whereas they are not orientated with $\langle\cos \theta\rangle \approx-0.059$ and -0.043 , respectively. For $\beta=75^{\circ}$, when the peak intensity of the Gaussian pulse is reached most of the states present a weak orientation, only the levels $|0,0, e\rangle$ and $|1,1, e\rangle$ have a large orientation for small values of $\mathrm{I}_{0}$.

These results indicate that with a 10 ns alignment pulse, strong dc fields and small inclination angles are required to reach a moderate orientation for excited states.

\section{B. Influence of the FWHM of the laser pulse}

For the same set of states as in the previous section, we analyze here how their directional properties depend on the FWHM of the Gaussian pulse. In Fig. 11 (a) and (b) we show $\langle\cos \theta\rangle$ at $t=0$ as a function of $\tau$ for $\beta=30^{\circ}$ and $45^{\circ}$, respectively. The static electric field is fixed to $\mathrm{E}_{\mathrm{s}}=300 \mathrm{~V} / \mathrm{cm}$, and the peak intensity to $\mathrm{I}_{0}=10^{12} \mathrm{~W} / \mathrm{cm}^{2}$.
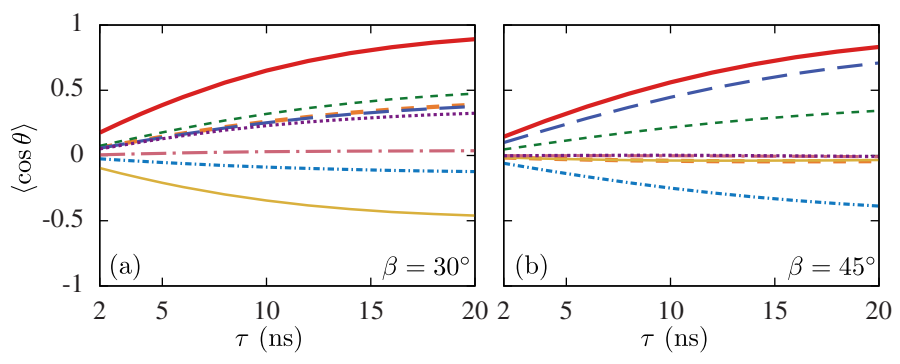

FIG. 11. (Color online) Expectation value $\langle\cos \theta\rangle$ at $t=0$ as a function of $\tau$. The field configurations are (a) $\beta=30^{\circ}$ and (b) $\beta=45^{\circ}$, with $\mathrm{I}_{0}=10^{12} \mathrm{~W} / \mathrm{cm}^{2}$ and $\mathrm{E}_{\mathrm{s}}=300 \mathrm{~V} / \mathrm{cm}$. The states and their labels are the same as in Fig. 10

The orientation of the ground state increases approaching the adiabatic limit as $\tau$ is increased, and it reaches it with a 50 ns pulse. We encounter several excited states 
presenting a moderate or weak orientation, and their $|\langle\cos \theta\rangle|$ monotonically increases as $\tau$ is enhanced, e.g., for $\beta=30^{\circ}$ the levels $|1,0, e\rangle,|1,1, e\rangle,|2,0, e\rangle,|2,1, e\rangle$, $|2,2, e\rangle$ and $|3,0, e\rangle$ and for $\beta=45^{\circ}|2,0, e\rangle,|2,1, e\rangle$ and $|2,2, e\rangle$. For all of them, a 20 ns pulse is not enough to achieve the adiabatic regime. In contrast, other excited levels present a very small, almost zero, orientation independently of the pulse duration. Some of these levels behave as dark states being strongly aligned but not oriented independently of the pulse duration, e.g., the $|3,2, e\rangle$ state has $\left\langle\cos ^{2} \theta\right\rangle=0.755$ and $|\langle\cos \theta\rangle|<0.04$ for $\beta=30^{\circ}$ and any value of $\tau$. An analogous behavior is found for the levels $|1,0, e\rangle,|1,1, e\rangle,|3,0, e\rangle$ and $|3,2, e\rangle$ at $\beta=45^{\circ}$. As described above, this phenomenon is due to the non-adiabatic dynamics at weak laser intensities when the levels of the $J$-manifold are driven apart, and it takes places even for $50 \mathrm{~ns}$ pulses.

\section{Influence of the electrostatic field strength}

In this section, we consider two inclination angles, and investigate the impact of the electrostatic field on the mixed-field orientation dynamics of the same states. Figures 12 (a) and (b) illustrate the behavior of $\langle\cos \theta\rangle$ at $t=0$ as a function of $\mathrm{E}_{\mathrm{s}}$ for $\beta=30^{\circ}$ and $45^{\circ}$, respectively. The laser pulse has $\tau=10 \mathrm{~ns}$ and $\mathrm{I}_{0}=10^{12} \mathrm{~W} / \mathrm{cm}^{2}$.

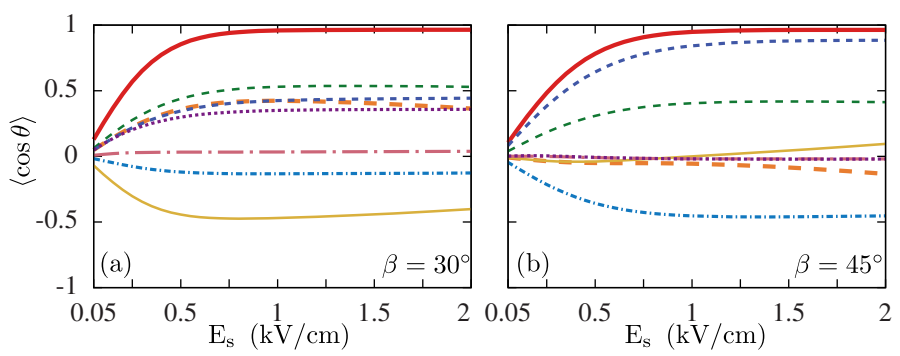

FIG. 12. (Color online) Expectation value of $\langle\cos \theta\rangle$ at $t=0$ as a function of $\mathrm{E}_{\mathrm{s}}$ for the field configurations $\tau=10 \mathrm{~ns}$, $\mathrm{I}_{0}=10^{12} \mathrm{~W} / \mathrm{cm}^{2}$, and (a) $\beta=30^{\circ}$ and (b) $\beta=45^{\circ}$. The labeling of the states is done as in Fig. 10

The ground state presents the largest orientation, which increases as $E_{\mathrm{s}}$ is enhanced, being strongly oriented for sufficiently large fields, e. g., $\langle\cos \theta\rangle>0.9$ for $\mathrm{E}_{\mathrm{s}} \geq 600 \mathrm{~V} / \mathrm{cm}$ and $\beta=30^{\circ}$. Regarding the excited states, their orientation strongly depends on the inclination angle. For $\beta=30^{\circ},|\langle\cos \theta\rangle|$ monotonically increases till it reaches a plateau-like behavior, and they show a moderate orientation. Indeed, for $\beta=30^{\circ}$, $\mathrm{I}_{0}=10^{12} \mathrm{~W} / \mathrm{cm}^{2}$ and $\mathrm{E}_{\mathrm{s}}=2 \mathrm{kV} / \mathrm{cm}$, we obtain at the maximum of the Gaussian pulse $\langle\cos \theta\rangle=-0.402$ and 0.365 for the states $|1,0, e\rangle$ and $|1,1, e\rangle$, respectively. For $\beta=45^{\circ}$, the level $|2,1, e\rangle$ presents a large orientation: $\langle\cos \theta\rangle>0.8$ for $\mathrm{E}_{\mathrm{s}} \gtrsim 800 \mathrm{~V} / \mathrm{cm}$. There are some darks states for $\beta=45^{\circ}$, which are not oriented even when dc fields of $2 \mathrm{kV} / \mathrm{cm}$ are used, e.g., $|3,0, e\rangle$ and $|3,2, e\rangle$.
For non-parallel fields, a strong dc field does not ensure a large orientation for excited rotational states. If the aim is a strongly oriented molecular ensemble, then this should be as pure as possible in the ground state.

In the Hamiltonian (3) the term $-\mu \mathrm{E}_{\mathrm{s}} \sin \beta \sin \theta \cos \phi$ is responsible for the mixing of states with different fieldfree magnetic quantum numbers. On the weak dc field regime, the mixing between these states is so small that $M$ could be considered as conserved, and this term could be neglected. By increasing $\mathrm{E}_{\mathrm{s}}$, this coupling between levels with different field-free $M$ becomes important, and this should affect the molecular dynamics. Thus, the questions that remain open is how important is the $X$ component of the electrostatic field to the dynamics, and for which electric field regime, we could only consider its $Z$-component $\mathbf{E}_{\mathrm{s}}=\mathrm{E}_{\mathrm{s}} \cos \beta \hat{Z}$.

As indicated above, even for tilted fields, the dynamics of the ground state can be described by a two state model. Its energy separation to the next state with $M \neq 0$ is of the order of $B$ and larger than the dc field coupling to these levels. Thus, for $\mathrm{E}_{\mathrm{s}} \lesssim 20 \mathrm{kV} / \mathrm{cm}$, the dynamics considering the dc field is equal to the one obtained when only its $Z$-component is included.

For the excited states, the answer to these questions depends on how the initial wave function, before the fields are switched on, is constructed. The first option is to proceed as indicated at the beginning of this section; the field-free $\beta \neq 0^{\circ}$ and $\beta=0^{\circ}$ wave functions are related by a rotation of $\beta$ around the $Y$-axis $|J, M, e\rangle=R_{Y}(\beta)|J, M, e\rangle^{0}$. In this case, for the level $|1,1, e\rangle$, some differences in its orientation are observed for $\mathrm{E}_{\mathrm{s}} \gtrsim 1 \mathrm{kV} / \mathrm{cm}$ with $|\langle\cos \theta\rangle|$ being larger if the two components of $\mathbf{E}_{\mathrm{s}}$ are considered. These differences are augmented as $\mathrm{E}_{\mathrm{s}}$ is increased, e.g., for a $10 \mathrm{~ns}$ laser pulse with $\mathrm{I}_{0}=1 \times 10^{12} \mathrm{~W} / \mathrm{cm}^{2}, \beta=45^{\circ}$ and $\mathrm{E}_{\mathrm{s}}=5 \mathrm{kV} / \mathrm{cm}$, we obtain at $t=0\langle\cos \theta\rangle=-0.629$ compared to $\langle\cos \theta\rangle=-0.019$ if only the $Z$-component of $\mathbf{E}_{\mathrm{s}}$ is included. By increasing $\mathrm{E}_{\mathrm{s}}$ this state will achieve an adiabatic dynamics only if both components of the static field are present. The second option is to construct the fieldfree $\beta \neq 0^{\circ}$ wave function equal to the field-free $\beta=0^{\circ}$ one. In this case, the results resemble those of the parallel field configuration taking into account $\cos \beta$ as scaling factor for the static field strength.

\section{Influence of the inclination of the fields}

The symmetries of the rotational Hamiltonian (1) see Sec. II and, therefore, the rotational dynamics strongly depend on the angle between the fields. In this section, we investigate in detail the impact of the inclination angle in the mixed-field orientation dynamics.

For the ground state $|0,0, e\rangle$, the orientation cosines $\langle\cos \theta\rangle$ and $\left\langle\cos \theta_{\mathrm{s}}\right\rangle$ are plotted in Fig. 13 as a function of $\beta$, together with the adiabatic results. For a weak dc field and strong laser field, the following relation $\left\langle\cos \theta_{\mathrm{s}}\right\rangle \approx\langle\cos \theta\rangle \cos \beta$ is satisfied within the adi- 
abatic limit. In $\left\langle\cos \theta_{\mathrm{s}}\right\rangle$ the term $\langle\sin \theta \cos \phi\rangle \sin \beta$ has been neglected, which can be done as far as the mixing between states with different field-free $M$ is very small. By increasing the electrostatic field strength, a regime would be encountered where this approximation does not hold any longer. An analogous relation is satisfied between the time-dependent orientation cosines of the ground state. For $\mathrm{I}_{0}=2 \times 10^{11} \mathrm{~W} / \mathrm{cm}^{2}$, its orientation $\langle\cos \theta\rangle$ shows a plateau-like behavior till $\beta=50^{\circ}$, which is very close to the adiabatic limit. By further increasing $\beta,\langle\cos \theta\rangle$ decreases and approaches to zero. For $\beta=90^{\circ}$, the states in a pendular doublet have different symmetry and are not coupled by the dc field, thus they might be strongly aligned but not oriented. For $\mathrm{I}_{0}=10^{12} \mathrm{~W} / \mathrm{cm}^{2},\langle\cos \theta\rangle$ monotonically decreases as $\beta$ is increased towards $90^{\circ}$, and its value is always smaller than for $\mathrm{I}_{0}=2 \times 10^{11} \mathrm{~W} / \mathrm{cm}^{2}$. For both laser fields, $\left\langle\cos \theta_{\mathrm{s}}\right\rangle$ decreases as $\beta$ is increased.

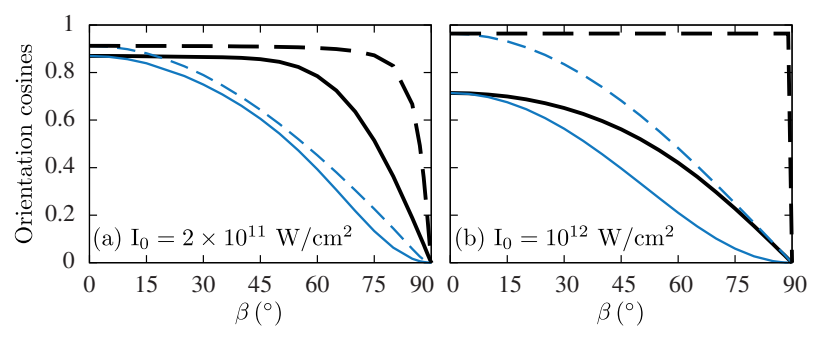

FIG. 13. (Color online) Expectation values $\langle\cos \theta\rangle$ (thick solid line) and $\left\langle\cos \theta_{\mathrm{s}}\right\rangle$ (thin solid line) at $t=0$ as a function of $\beta$ for the ground state. The peak intensities are (a) $I_{0}=$ $2 \times 10^{11} \mathrm{~W} / \mathrm{cm}^{2}$ and (b) $\mathrm{I}_{0}=10^{12} \mathrm{~W} / \mathrm{cm}^{2}$. The adiabatic results for $\langle\cos \theta\rangle$ (thick dashed line) and $\left\langle\cos \theta_{\mathrm{s}}\right\rangle$ (thin dashed line) are also presented. The FWHM of the laser pulse is fixed to $\tau=10 \mathrm{~ns}$ and the dc field to $\mathrm{E}_{\mathrm{s}}=300 \mathrm{~V} / \mathrm{cm}$.

In Figs. 14 (a), (b), (c) and (d), we present the orientation cosine of the pairs $|0,0, e\rangle-|1,0, e\rangle,|1,1, e\rangle-|2,1, e\rangle$, $|2,2, e\rangle-|3,2, e\rangle$ and $|2,0, e\rangle-|3,0, e\rangle$, respectively, as a function $\beta$. The static field strength is $\mathrm{E}_{\mathrm{s}}=300 \mathrm{~V} / \mathrm{cm}$ and we consider two Gaussian pulses of 10 ns FWHM and peak intensities $\mathrm{I}_{0}=2 \times 10^{11} \mathrm{~W} / \mathrm{cm}^{2}$ and $10^{12} \mathrm{~W} / \mathrm{cm}^{2}$. Due to the complicated field-dressed dynamics of excited states for $0^{\circ}<\beta<90^{\circ}$ with contributions from several pendular pairs, in $\left\langle\cos \theta_{\mathrm{s}}\right\rangle$ the term $\langle\sin \theta \cos \phi\rangle$ cannot be neglected. Then, the simple relation $\left\langle\cos \theta_{\mathrm{s}}\right\rangle \approx$ $\langle\cos \theta\rangle \cos \beta$ does not hold for these levels.

Based on the adiabatic theory, the ground state and the level $|1,0, e\rangle$, should present the same orientation but with opposite directions. However, this is only satisfied for $\beta=0^{\circ}$. Due to the non-adiabatic effects at weak laser intensities, its $|\langle\cos \theta\rangle|$ is smaller than the corresponding value of $|0,0, e\rangle$ for $0<\beta<90^{\circ}$. For the second doublet, cf. Fig. 14 (b), $\langle\cos \theta\rangle$ oscillates as $\beta$ is varied, and the orientation even changes its direction. Both states could present a moderate orientation at a certain value of $\beta$. The pendular regime is not achieved by the third and fourth pairs with a $10 \mathrm{~ns}$ laser pulse and $\mathrm{I}_{0}=2 \times 10^{11} \mathrm{~W} / \mathrm{cm}^{2}$, and their orientation is either zero

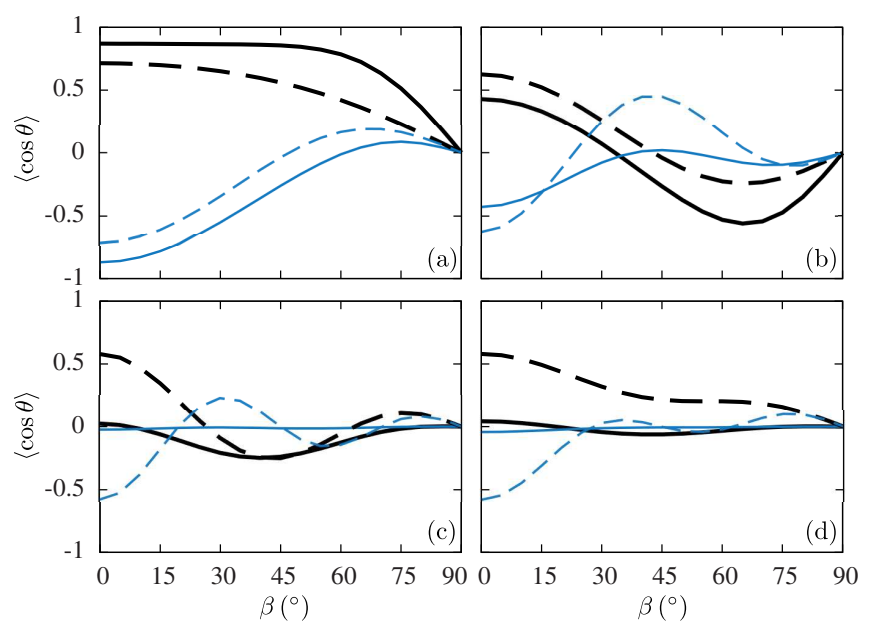

FIG. 14. (Color online) Expectation value $\langle\cos \theta\rangle$ at $t=0$ as a function of $\beta$ for the states (a) $|0,0, e\rangle$ (black), and $|1,0, e\rangle$ (blue), (b) $|1,1, e\rangle$ (black), and $|2,1, e\rangle$ (blue), (c) $|2,0, e\rangle$ (black), and $|3,0, e\rangle$ (blue), (d) $|2,2, e\rangle$ (black), and $|3,2, e\rangle$ (blue). The field configuration is $\mathrm{E}_{\mathrm{s}}=300 \mathrm{~V} / \mathrm{cm}$ and $\mathrm{I}_{0}=2 \times 10^{11} \mathrm{~W} / \mathrm{cm}^{2}$ (solid lines) and $10^{12} \mathrm{~W} / \mathrm{cm}^{2}$ (dashed lines). The FWHM of the laser pulse is fixed to $\tau=10 \mathrm{~ns}$.

or very small independently of $\beta$. For $\mathrm{I}_{0}=10^{12} \mathrm{~W} / \mathrm{cm}^{2}$ and $\beta=0^{\circ}$, these four states show a moderate orientation, which is reduced for any other angle, being small for $\beta \gtrsim 60^{\circ}$. At the strong peak intensity $\mathrm{I}_{0}=10^{12} \mathrm{~W} / \mathrm{cm}^{2}$, in all pendular doublets one of the two levels presents the dark behavior with respect to the mixed-field orientation dynamics at a certain angle $\beta$.

These results show that if the molecular beam is rotationally cold, a small inclination angle will optimize the degree of orientation observed in the experiment.

\section{ROTATIONAL DYNAMICS ONCE THE LASER PULSE IS TURNED ON}

Let us investigate the dynamics for $t>0$ assuming that the laser peak intensity, reached at $t=0$, and the dc field strength are kept constant for $t>0$; i. e., $\mathrm{I}(t)=\mathrm{I}_{0}$ and $\mathrm{E}_{\mathrm{s}}(t)=\mathrm{E}_{\mathrm{s}}$ for $t>0$. At $t=0$, the time-dependent wave function can be expressed in terms of the corresponding adiabatic basis. Since the Hamiltonian is timeindependent for $t>0$, the contribution of each adiabatic state remains constant as $t$ is increased. For a certain state $|\gamma\rangle$, the expectation value of an operator $\hat{A}$ in this adiabatic basis reads as

$$
\begin{aligned}
\langle\hat{A}\rangle & =\sum_{j}\left|C_{j}(0)\right|_{\mathrm{p}}^{2}\left\langle\gamma_{j}|\hat{A}| \gamma_{j}\right\rangle_{\mathrm{p}} \\
& +2 \sum_{j<k}\left|C_{j}(0)\right|\left|C_{k}(0)\right|_{\mathrm{p}}\left\langle\gamma_{j}|\hat{A}| \gamma_{k}\right\rangle_{\mathrm{p}} \cos \left(\frac{\Delta E_{j k} t}{\hbar}+\delta_{j k}\right)
\end{aligned}
$$

with $C_{j}(0)$ being the weight at $t=0$ of the adiabatic state $\left|\gamma_{j}\right\rangle_{\mathrm{p}}$ to the wave function of $|\gamma\rangle, \Delta E_{j k}$ the energy 
splitting between the adiabatic levels $\left|\gamma_{j}\right\rangle_{\mathrm{p}}$ and $\left|\gamma_{k}\right\rangle_{\mathrm{p}}$, and $\delta_{j k}$ the phase difference of $C_{j}(0)$ and $C_{k}(0)$.

Based on the results presented above, the timedependent wave function could have contributions from: i) only the adiabatic levels forming a pendular doublet, or ii) several adiabatic levels from at least two pendular doublets. All the states for $\beta=0^{\circ}$, and the ground state for $0^{\circ} \leq \beta<90^{\circ}$ could belong to the first case. Whereas, the second one refers to all excited states when $0^{\circ}<\beta<90^{\circ}$, unless the static field is very strong.
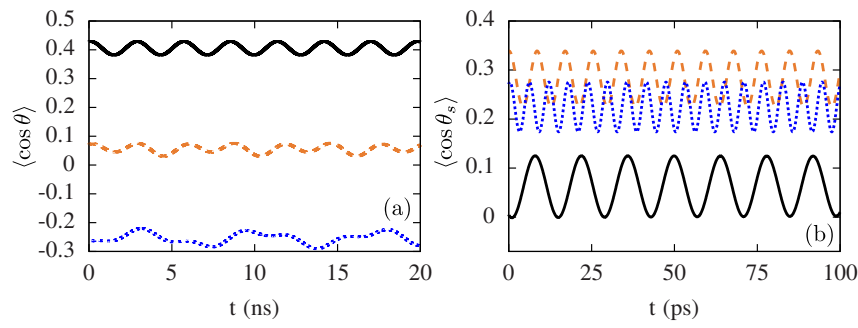

FIG. 15. (Color online) Orientation cosines once the peak intensity and dc field strength are kept constant for $t>0$. For the state $|1,1, e\rangle$, (a) expectation value $\langle\cos \theta\rangle$ with $\mathrm{I}_{0}=2 \times 10^{11} \mathrm{~W} / \mathrm{cm}^{2}$ and $\beta=0^{\circ}$ (solid), $30^{\circ}$ (dashed) and $45^{\circ}$ (dotted); and (b) $\left\langle\cos \theta_{\mathrm{s}}\right\rangle$ for $\beta=30^{\circ}$ and $\mathrm{I}_{0}=2 \times 10^{11} \mathrm{~W} / \mathrm{cm}^{2}$ (solid), $5 \times 10^{11} \mathrm{~W} / \mathrm{cm}^{2}$ (dashed) and $10^{12} \mathrm{~W} / \mathrm{cm}^{2}$ (dotted). The dc field is $E_{\mathrm{s}}=300 \mathrm{~V} / \mathrm{cm}$.

Let us first analyze the case when the dynamics takes place within a pendular doublet. If the adiabatic states are not fully oriented, the coupling term in Eq. (7) is non-zero and this expectation value oscillates for $t>0$ with the frequency equal to the energy splitting of the corresponding pendular doublet. For the $|1,1, e\rangle$ state, this behavior is shown for the time evolution of $\langle\cos \theta\rangle$ in Fig. 15 (a), with $\mathrm{I}_{0}=2 \times 10^{11} \mathrm{~W} / \mathrm{cm}^{2}, \mathrm{E}_{\mathrm{s}}=300 \mathrm{~V} / \mathrm{cm}$ and $\beta=0^{\circ}$. An analogous behavior is obtained for the ground state and $0^{\circ}<\beta<90^{\circ}$. By further increasing the peak intensity, the orientation of the adiabatic states increases, the coupling terms are reduced approaching zero, and these regular oscillations will disappear.

When two pendular doublets participate in the dynamics, this oscillatory behavior becomes irregular, because the frequencies associated with the energy separations within each pendular doublet and between two of them do not form a commensurable set. As an example, we show in Fig. 15 (a) these irregular oscillations of $\langle\cos \theta\rangle$ for $|1,1, e\rangle$ with $\beta=30^{\circ}$ and $45^{\circ}, \mathrm{E}_{\mathrm{s}}=300 \mathrm{~V} / \mathrm{cm}$ and $\mathrm{I}_{0}=2 \times 10^{11} \mathrm{~W} / \mathrm{cm}^{2}$. By increasing $\mathrm{I}_{0}$, the dynamics of this state still has contributions from different pendular doublets, but the two states in a pendular pair are not populated. As a consequence, the coupling terms are reduced and the oscillation decreases or even disappears.

For $0^{\circ}<\beta<90^{\circ}$, the time evolution of $\left\langle\cos \theta_{\mathrm{s}}\right\rangle$ is dominated by the couplings of adiabatic levels from doublets with $|\Delta M| \approx 1$. This is illustrated in Fig. 15 (b), for the state $|1,1, e\rangle, \mathrm{I}_{0}=2 \times 10^{11} \mathrm{~W} / \mathrm{cm}^{2}, 5 \times 10^{11} \mathrm{~W} / \mathrm{cm}^{2}$ and $10^{12} \mathrm{~W} / \mathrm{cm}^{2}$ and $\beta=30^{\circ}$. Independently of $\mathrm{I}_{0}$, in this time scale $\left\langle\cos \theta_{\mathrm{s}}\right\rangle$ oscillates with the largest frequency given by the energy gap between of the two pendular doublets involved, which is similar for the three peak intensities. On a larger time scale, the frequencies due to the states in a doublet will modulate the oscillations of $\left\langle\cos \theta_{\mathrm{s}}\right\rangle$ in the weak field regime.

\section{SWITCHING ON THE LASER PULSE FIRST: ORIENTATION OF THE ALIGNED PENDULAR STATES.}

In previous sections, the field configuration was based on the mixed-field orientation experiments [22, 24, 26]. Here, we investigate the molecular dynamics when the temporal order of the fields is inverted: the Gaussian pulse is switched on first, its peak intensity is kept constant, and then the static electric field is turned on. While the laser field is switched on, the pendular doublets of quasi-degenerate states with opposite parity are formed. This process is adiabatic [41, 42], and for a sufficiently large peak intensity, these two levels are strongly aligned but not oriented. By turning on the static field, these states have the same symmetry and they should be oriented due to their interaction with this field. For this field configuration, we check now the validity of the adiabatic predictions [18, 19] by comparing them to a time dependent analysis.

The peak intensity of the Gaussian pulse is reached at $t=0$ and kept constant afterwards. At this point, if $I_{0}$ is large enough, the energy gap between the states in a pendular doublet is much smaller than the energy gap with the neighboring doublet. Then, for a certain pendular level, its rotational dynamics for $t>0$, i.e., when the static field is switched on, could be approximated by a two-state model involving the two levels forming the corresponding pendular doublet [19]. At $t=0$, i.e., $\mathrm{E}_{\mathrm{s}}(0)=0$ and $\mathrm{I}(0)=\mathrm{I}_{0}$, the pendular states are $\left|\psi_{l}\right\rangle$ with $l=e$ and $o$ indicating even or odd parity. Under this approximation, we assume that the levels $\left|\psi_{r}\right\rangle=\frac{1}{\sqrt{2}}\left(\left|\psi_{e}\right\rangle+\left|\psi_{o}\right\rangle\right)$ and $\left|\psi_{w}\right\rangle=\frac{1}{\sqrt{2}}\left(\left|\psi_{e}\right\rangle-\left|\psi_{o}\right\rangle\right)$ are right- and wrong-way oriented, respectively. The twostate-model Hamiltonian yields as

$$
H(t)=\left(\begin{array}{cc}
0 & -\mu v_{\mathrm{s}} t\left\langle\cos \theta_{\mathrm{s}}\right\rangle_{e o} \\
-\mu v_{\mathrm{s}} t\left\langle\cos \theta_{\mathrm{s}}\right\rangle_{e o} & \Delta E
\end{array}\right),
$$

where we have taken $\mathrm{E}_{\mathrm{s}}(t)=v_{\mathrm{s}} t$ with $v_{\mathrm{s}}=\mathrm{E}_{\mathrm{s}} / T_{0}$ and $T_{0}$ being the switching on speed and time, respectively. This time $T_{0}$ is chosen so that if these states are exposed only to this field, the turning-on process is adiabatic. Note that we have taken $\left\langle\psi_{e}|H| \psi_{e}\right\rangle=0,\left\langle\psi_{o}|H| \psi_{o}\right\rangle=\Delta E$, and $\left\langle\psi_{e}|H| \psi_{o}\right\rangle=\left\langle\psi_{o}|H| \psi_{e}\right\rangle=-\mu v_{\mathrm{s}} t\left\langle\psi_{e}\left|\cos \theta_{\mathrm{s}}\right| \psi_{o}\right\rangle=$ $-\mu v_{\mathrm{s}} t\left\langle\cos \theta_{\mathrm{s}}\right\rangle_{e o}$. The time-dependent Schrödinger equation associated to this Hamiltonian admits a scaling factor. That is, when the dynamics is adiabatic using $v_{\mathrm{s}}$ for a pendular doublet with energy splitting $\Delta E$ at $\mathrm{I}(0)=\mathrm{I}_{0}$, then, for a peak intensity $\mathrm{I}_{0}^{\prime}$ and $\Delta E^{\prime}=k \Delta E$, the dynamics is adiabatic for $v_{\mathrm{s}}^{\prime}=k^{2} v_{\mathrm{s}}$. 

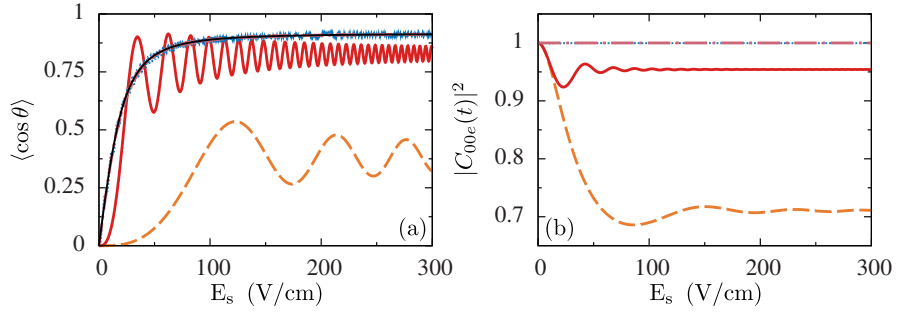

FIG. 16. (Color online) For the ground state, (a) expectation value $\langle\cos \theta\rangle$ and (b) weight of the adiabatic ground state on its time-dependent wave function as a function of $\mathrm{E}_{\mathrm{s}}(t)$, for turning on speeds $v_{\mathrm{s}}=10^{10}$ (orange dashed), $10^{9}$ (red solid), $10^{8}$ (blue dotted) and $10^{7} \mathrm{~V} / \mathrm{scm}$ (pink dot-dashed), and adiabatic results (thin solid) The fields are parallel and the Gaussian pulse has $\tau=10 \mathrm{~ns}$ and $\mathrm{I}_{0}=2 \times 10^{11} \mathrm{~W} / \mathrm{cm}^{2}$.

For the sake of simplicity, we focus on the ground state in a parallel-field configuration. For several switching on speeds, Fig. 16 (a) and (b) display the directional cosine and the population of the adiabatic ground state, respectively, as a function of $\mathrm{E}_{\mathrm{s}}(t)$. The Gaussian pulse has $10 \mathrm{~ns}$ FWHM and peak intensity $\mathrm{I}_{0}=2 \times 10^{11} \mathrm{~W} / \mathrm{cm}^{2}$. Before the dc field is turned on, the alignment of the ground state is $\left\langle\cos ^{2} \theta\right\rangle=0.845$, the energy separation within this pendular pair is $\Delta E \approx 5.36 \times 10^{-4} \mathrm{~cm}^{-1}$, and there are $2.4 \mathrm{~cm}^{-1}$ to the next pendular doublet. For $\mathrm{E}_{\mathrm{s}}=1 \mathrm{~V} / \mathrm{cm}$, the coupling term is $\mu\langle\cos \theta\rangle_{e o} \mathrm{E}_{\mathrm{s}}=$ $1.09 \times 10^{-5} \mathrm{~cm}^{-1}$ with $\langle\cos \theta\rangle_{e o}=0.915$. In an adiabatic picture, the energy gap $\Delta E$ can not be neglected, and as $\mathrm{E}_{\mathrm{S}}(t)$ is increased the energy of the ground state does not increase linearly with $\mathrm{E}_{\mathrm{s}}[19]$. For $v_{\mathrm{s}}=10^{10} \mathrm{~V} / \mathrm{scm}$, the adiabatic parameter $\eta \approx 1$, the rotational dynamics is non-adiabatic and there is a population transfer between the two states in this doublet. We note that for this process, the adiabatic parameter $\eta$ is defined as in Eq. (6) but replacing the laser field interaction $H_{\mathrm{L}}(t)(4)$ by the dipole term $H_{\mathrm{S}}(t)(3)$. The ground state presents a moderate orientation bellow the adiabatic limit due to the contributions of the adiabatic states $|0,0, e\rangle_{\mathrm{p}}$ and $|1,0, e\rangle_{\mathrm{p}},\left|C_{00 e}(t)\right|^{2}$ decreases until a minimum value showing a smooth oscillation afterwards, cf. Fig. 16 (b). Due to the coupling term, $\langle\cos \theta\rangle$ oscillates as $\mathrm{E}_{\mathrm{S}}(t)$ is increased, and its frequency is equal to the energy separation between the adiabatic levels $|0,0, e\rangle_{\mathrm{p}}$ and $|1,0, e\rangle_{\mathrm{p}}$. A similar behavior is observed for $10^{9} \mathrm{~V} / \mathrm{scm}$, but the orientation of the ground state oscillates around a value closer to the adiabatic limit because the process is more adiabatic and $\left|C_{00 e}(t)\right|^{2} \approx 0.956$ for $\mathrm{E}_{\mathrm{s}}(t) \gtrsim 100 \mathrm{~V} / \mathrm{cm}$. For $v_{\mathrm{s}}=10^{8}$ and $10^{7} \mathrm{~V} / \mathrm{scm}$, the dynamics can be considered as adiabatic, with $\left|C_{00 e}(t)\right|^{2}$ being larger than 0.999. However, for $v_{\mathrm{s}}=10^{8},\langle\cos \theta\rangle$ still oscillates around the adiabatic value.

By increasing the peak intensity of the laser pulse, the energy splitting of the levels in a pendular doublet is decreased, but their coupling due to the dc field is not significantly modified. Thus, the rotational dynamics becomes more diabatic, and larger turning-on times are needed to achieve the adiabatic limit. For $\mathrm{I}_{0}=5 \times 10^{11} \mathrm{~W} / \mathrm{cm}^{2}$, the ground state is separated by $\Delta E \approx 7.7 \times 10^{-7} \mathrm{~cm}^{-1}$ from $|1,0, e\rangle_{\mathrm{p}}$, and by $3.9 \mathrm{~cm}^{-1}$ from the next pendular doublet. The coupling due to the dc field is $\mu\langle\cos \theta\rangle_{e o} \mathrm{E}_{\mathrm{s}}=1.13 \times 10^{-5} \mathrm{~cm}^{-1}$ for $\mathrm{E}_{\mathrm{s}}=1 \mathrm{~V} / \mathrm{cm}$ and with $\langle\cos \theta\rangle_{e o}=0.948$. According to the scaling law of the time-dependent Schrödinger equation, this process would be adiabatic for a speed of $v_{\mathrm{s}} \lesssim 20 \mathrm{~V} / \mathrm{scm}$.

For $\mathrm{I}_{0}=10^{12} \mathrm{~W} / \mathrm{cm}^{2}$ and the ground state, we find $\Delta E \approx 3.3 \times 10^{-10} \mathrm{~cm}^{-1}, 5.7 \mathrm{~cm}^{-1}$ to the second doublet, and $\mu\langle\cos \theta\rangle_{e o} \mathrm{E}_{\mathrm{s}}=1.18 \times 10^{-5} \mathrm{~cm}^{-1}$ for $\mathrm{E}_{\mathrm{s}}=$ $1 \mathrm{~V} / \mathrm{cm}$ and with $\langle\cos \theta\rangle_{e o}=0.964$. Within an adiabatic framework, as $\mathrm{E}_{\mathrm{S}}$ is increased the ground state energy can be approximated by the pseudo-first-order Stark linear effect $\left|\mu \mathrm{E}_{\mathrm{s} p}\langle 0,0, e|\cos \theta| 1,0, e\rangle_{\mathrm{p}}\right|$ [19]. Note that $\Delta E$ is smaller than the dc filed coupling even for $\mathrm{E}_{\mathrm{s}} \approx 10^{-4} \mathrm{~V} / \mathrm{cm}$. Based on the scaling law of the twostate model Schrödinger equation, the dc field should be turned on very slowly, $v_{\mathrm{s}} \lesssim 10^{-2} \mathrm{~V} / \mathrm{scm}$, to achieve the adiabatic limit. For larger turning-on speeds, the dynamics is so diabatic that the $|0,0, e\rangle$ wave function does not change, and its projections on the adiabatic states $|0,0, e\rangle_{\mathrm{p}}$ and $|1,0, e\rangle_{\mathrm{p}}$ are close to the field-free values even for $v_{\mathrm{s}} \approx 10^{5} \mathrm{~V} / \mathrm{scm}$.

\section{CONCLUSIONS}

In this work, we have investigated the mixed-field orientation dynamics of linear molecules. The richness and variety of the field-dressed rotational dynamics has been illustrated by analyzing in detail the directional properties of several low-lying states. In particular, we have explored the degree of orientation as the peak intensity and FWHM of the Gaussian pulse, the electrostatic field strength and the angle between both fields are varied.

By considering prototypical field configurations used in current mixed-field orientation experiments, we have proven that the assumption of a fully adiabatic dynamics is incorrect. For parallel fields, a non-adiabatic transfer of population takes place when the quasi-degenerated pendular doublets are formed as the laser intensity is increased. As a consequence, the time-dependent results for the degree of orientation are smaller than the predictions of the adiabatic theory. Using current available experimental peak intensities, longer laser pulses or stronger static fields will increase the degree of orientation even for highly excited states. In particular, we have provided the field parameters under which the mixedfield orientation dynamics will be fully adiabatic. We have also shown that the field-dressed dynamics is more complicated if both fields are tilted. Apart from the nonadiabatic effects when the pendular doublets are formed, at weak laser intensities there is also population transfer due to the splitting of the states within a $J$-manifold having now the same symmetry. For non-parallel fields, we have shown that the ground state is strongly oriented, whereas excited states might only present a moderate ori- 
entation, and, furthermore, some of them could behave as dark states to the mixed-field orientation dynamics. The requirements for an adiabatic dynamics are now more difficult to satisfy for excited levels than for the ground state. Again, we have indicated the field configuration that will give rise to an adiabatic mixed-field-orientation. If the peak intensity is kept constant after turning on the pulse, we have shown that the orientation of the states might oscillate with time due to the non-adiabatic dynamics. Finally, we have investigated the molecular dynamics when the temporal order of the fields is inverted. We have shown that once the ground state is adiabatically aligned, the switching on of the dc field has to be very slow to achieve a significant orientation.

Although our study is restricted to the OCS molecule, we stress that the above-observed physical phenomena are expected to occur in many other polar molecules. In- deed, the Hamiltonian can be rescaled, and the above results used to describe another polar linear molecule. In addition, due the complexity of their rotational level structure of asymmetric tops, these non-adiabatic effects should have a negative impact in their mixed-field orientation experiments [22, 25].

\section{ACKNOWLEDGMENTS}

We would like to thank B. Friedrich, J. Küpper, J. H. Nielsen, P. Schmelcher and H. Stapelfeldt for fruitful discussions. Financial support by the Spanish project FIS2011-24540 (MICINN), the Grants FQM-2445 and FQM-4643 (Junta de Andalucía), and Andalusian research group FQM-207 is gratefully appreciated. J.J.O. acknowledges the support of ME under the program FPU.
[1] P. R. Brooks, Science 193, 11 (1976).

[2] P. R. Brooks and E. M. Jones, J. Chem. Phys. 45, 3449 (1966).

[3] H. J. Loesch and J. Möller, J. Chem. Phys. 97, 9016 (1992).

[4] F. J. Aoiz, B. Friedrich, V. J. Herrero, V. S. Rábanos, and J. E. Verdasco, Chem. Phys. Lett. 289, 132 (1998).

[5] V. Aquilanti, M. Bartolomei, F. Pirani, D. Cappelletti, and F. Vecchiocattivi, Phys. Chem. Chem. Phys 7, 291 (2005).

[6] C. Z. Bisgaard, O. J. Clarkin, G. Wu, A. M. D. Lee, O. Gessner, C. C. Hayden, and A. Stolow, Science 323, 1464 (2009).

[7] L. Holmegaard, J. L. Hansen, L. Kalhoj, S. L. Kragh, H. Stapelfeldt, F. Filsinger, J. Küpper, G. Meijer, D. Dimitrovski, M. Abu-samha, et al., Nat. Phys. 6, 428 (2010).

[8] J. L. Hansen, H. Stapelfeldt, D. Dimitrovski, M. Abusamha, C. P. J. Martiny, and L. B. Madsen, Phys. Rev. Lett. 106, 073001 (2011).

[9] E. Frumker, C. T. Hebeisen, N. Kajumba, H. J. W. J. B. Bertrand and, M. Spanner, D. M. Villeneuve, A. Naumov, and P. Corkum, arXiv:1205.4199v1.

[10] P. M. Kraus, S. Vlajkovic, A. Rupenyan, and H. J. Wörner, Phys. Rev. Lett. accepted (2012).

[11] S. Stolte, Atomic and Molecular Beam Methods (Oxford University Press, New York, 1988).

[12] H. J. Loesch and A. Remscheid, J. Chem. Phys. 93, 4779 (1990).

[13] B. Friedrich and D. R. Herschbach, Nature 353, 412 (1991).

[14] B. Friedrich, D. P. Pullman, and D. R. Herschbach, J. Phys. Chem. 95, 8118 (1991).

[15] P. A. Block, E. J. Bohac, and R. E. Miller, Phys. Rev. Lett. 68, 1303 (1992).

[16] B. Friedrich, H.-G. Rubahn, and N. Sathyamurthy, Phys. Rev. Lett. 69, 2487 (1992).

[17] A. Slenczka, B. Friedrich, and D. Herschbach, Phys. Rev. Lett. 72, 1806 (1994).
[18] B. Friedrich and D. R. Herschbach, J. Chem. Phys. 111, 6157 (1999).

[19] B. Friedrich and D. Herschbach, J. Phys. Chem. A 103, 10280 (1999).

[20] H. Sakai, S. Minemoto, H. Nanjo, H. Tanji, and T. Suzuki, Phys. Rev. Lett. 90, 083001 (2003).

[21] U. Buck and M. Fárník, Int. Rev. Phys. Chem. 25, 583 (2006).

[22] L. Holmegaard, J. H. Nielsen, I. Nevo, H. Stapelfeldt, F. Filsinger, J. Küpper, and G. Meijer, Phys. Rev. Lett. 102, 023001 (2009).

[23] O. Ghafur, A. Rouzee, A. Gijsbertsen, W. K. Siu, S. Stolte, and M. J. J. Vrakking, Nat Phys 5, 289 (2009).

[24] F. Filsinger, J. Küpper, G. Meijer, L. Holmegaard, J. H. Nielsen, I. Nevo, J. L. Hansen, and H. Stapelfeldt, J. Chem. Phys. 131, 064309 (2009).

[25] J. J. Omiste, M. Gärttner, P. Schmelcher, R. GonzálezFérez, L. Holmegaard, J. H. Nielsen, H. Stapelfeldt, and J. Küpper, Phys. Chem. Chem. Phys. 13, 18815 (2011).

[26] J. H. Nielsen, H. Stapelfeldt, J. Küpper, B. Friedrich, J. J. Omiste, and R. González-Férez, Phys. Rev. Lett. 108, 193001 (2012).

[27] C. M. Dion, A. Keller, O. Atabek, and A. D. Bandrauk, Phys. Rev. A 59, 1382 (1999).

[28] N. E. Henriksen, Chem. Phys. Lett. 312, 196 (1999).

[29] M. D. Feit, J. A. Fleck Jr., and A. Steiger, J. Comp. Phys. 47, 412 (1982).

[30] Z. Bačic̀ and J. C. Light, Annu. Rev. Phys. Chem. 40, 469 (1989).

[31] G. C. Corey and D. Lemoine, J. Chem. Phys. 97, 4115 (1992).

[32] A. R. Offer and G. G. Balint-Kurti, J. Chem. Phys. 101, 10416 (1994).

[33] P. Sánchez-Moreno, R. González-Férez, and P. Schmelcher, Phys. Rev. A 76, 053413 (2007).

[34] M. Härtelt and B. Friedrich, J. Chem. Phys. 128, 224313 (2008).

[35] L. B. Ballentine, Quantum Mechanics: A Modern Development (World Scientific, Singapore, 1998). 
[36] B. Friedrich, N. Nahler, and U. Buck, J. Mod. Opt. 50, 2677 (2003).

[37] M. D. Poulsen, T. Ejdrup, H. Stapelfeldt, E. Hamilton, and T. Seideman, Phys. Rev. A 73, 033405 (2006).

[38] Y. Sugawara, A. Goban, S. Minemoto, and H. Sakai, Phys. Rev. A 77, 031403(R) (2008).

[39] M. Muramatsu, M. Hita, S. Minemoto, and H. Sakai, Phys. Rev. A 79, 011403(R) (2009).
[40] R. N. Zare, Angular Momentum: Understanding Spatial Aspects in Chemistry and Physics (John Wiley and Sons, New York, 1988).

[41] J. Ortigoso, M. Rodríguez, M. Gupta, and B. Friedrich, J. Chem. Phys. 110, 3870 (1999).

[42] S. S. Viftrup, V. Kumarappan, S. Trippel, H. Stapelfeldt, E. Hamilton, and T. Seideman, Phys. Rev. Lett. 99, 143602 (2007). 